\title{
Aromatic hydroxylation by cytochrome P450: Mechanism and substituent effects
}

Christine M. Bathelt, Lars Ridder, Adrian J. Mulholland, and Jeremy N. Harvey School of Chemistry, University of Bristol, Cantock's Close, Bristol BS8 1TS, U.K.

\section{Supporting Information}

Contents:

Computational Methods

Table S1: computed gas-phase energies relating to the energy profile for reaction of benzene with Compound I.

Table S2: UB3LYP/BS I computed activation energies for addition of Compound I to the meta position of some substituted aromatics.

Table S3: List of computed activation and reaction energies for addition of Compound I to the para position of some substituted aromatics. Hammett parameters for the substituents are also provided. Plots of UB3LYP/BS II//UB3LYP/BS I calculated activation energies vs. Hammett parameters are provided as Figs S1 and S2.

Table S4: computed spin- and charge- densities on the transition states for addition of Compound I to the para position of some substituted aromatics.

Table S5: computed activation energies for addition of Compound I to the para position of some substituted aromatics in the presence of a continuum electrostatic environment.

Optimized Geometries and Total energies of all species.

\section{Computational Methods}

All calculations have been carried out using the Jaguar 4.2 software (Schrödinger, Inc., Portland, OR, 1991-2002) using the unrestricted hybrid density functional UB3LYP. Geometry optimizations were performed using the standard Los Alamos effective core potential and associated triple-zeta basis set on Fe (LACV3P basis), and the 6-31G* basis on all other atoms (BS I). Additional single point energy calculations were carried out at the optimized geometries using a larger basis set (BS II), which uses the same LACV3P basis on Fe, but the larger 6$311+\mathrm{G}^{* *}$ basis on other atoms. Charge and spin-density analysis was carried out with NBO. Single point energy calculations were carried out for some systems in the presence of a polarizable continuum (see Table S5). 
Table S1: B3LYP total (in hartrees) and relative (in $\mathrm{kcal} / \mathrm{mol}$ ) energies for addition of Compound I to benzene and subsequent rearrangement of the electrophilic intermediate to the ketone and epoxide product, respectively. Relative energies are given with respect to the reactants (Compound I + benzene).

\begin{tabular}{ccccc}
\hline & $\mathrm{E}_{\text {tot }}(\mathrm{BSI})$ & $\mathrm{E}_{\text {tot }}(\mathrm{BSII})$ & $\mathrm{E}_{\text {rel }}(\mathrm{BSI})$ & $\mathrm{E}_{\text {rel }}(\mathrm{BSII})$ \\
Benzene+CompoundI & -1857.37230 & -1857.75797 & 0.0 & 0.0 \\
TS & -1857.34187 & -1857.73028 & 19.1 & 17.4 \\
radical-like adduct & -1857.35547 & $/$ & 10.6 & $/$ \\
electrophilic adduct & -1857.36509 & -1857.75530 & 4.5 & 1.7 \\
NIH shift TS & -1857.36362 & -1857.75912 & 5.4 & 0.8 \\
NIH shift product & -1857.43543 & -1857.82561 & -39.6 & -42.4 \\
epoxide forming TS & -1857.35289 & -1857.74250 & 12.2 & 9.7 \\
epoxide product & -1857.38618 & -1857.77614 & -8.7 & -11.4 \\
\hline
\end{tabular}

Table S2: B3LYP activation energies (in $\mathrm{kcal} / \mathrm{mol}$ ) for addition of Compound I to metasubstituted benzenes calculated with basis set BSI.

\begin{tabular}{cr}
\hline substituent & $\mathrm{E}_{\mathrm{a}}(\mathrm{BSI})$ \\
$\mathrm{H}$ & 19.1 \\
$\mathrm{~m}-\mathrm{Cl}$ & 19.5 \\
$\mathrm{~m}-\mathrm{OMe}$ & 20.0 \\
$\mathrm{~m}-\mathrm{CN}$ & 18.9 \\
$\mathrm{~m}-\mathrm{NO}_{2}$ & 19.1 \\
\hline
\end{tabular}

Table S3: UB3LYP computed activation energies and heats of reaction (in $\mathrm{kcal} / \mathrm{mol}$ ) for paraaddition of Compound I model to substituted benzenes calculated with basis sets BSI and BSII. Also shown are the radical ( rad, see Dinctuerk, S.; Jackson, R. A. J. Chem. Soc., Perkin Trans. 2 1981, 1127-1131), cationic ( ${ }^{+}$, see Taylor, R. Electrophilic aromatic substitution; Wiley: Chichester, 1990; 458-463), and combined ( $\mathrm{rad}-0.3^{+}$) Hammett parameters used to plot the correlation Figs. 2, S1 and S2.

\begin{tabular}{ccccrrrc}
\hline substituent & $\mathrm{E}_{\mathrm{a}}(\mathrm{BSI})$ & $\mathrm{E}_{\mathrm{a}}(\mathrm{BSII})$ & $\mathrm{H}(\mathrm{BSI})$ & $\mathrm{H}(\mathrm{BSII})$ & $\mathrm{rad}$ & + & $\mathrm{rad}-0.3+$ \\
\hline $\mathrm{H}$ & 19.1 & 17.4 & 4.5 & 1.7 & 0.09 & 0.0 & 0.09 \\
$\mathrm{~F}$ & 18.0 & 16.3 & 2.2 & -0.2 & 0.12 & -0.075 & 0.093 \\
$\mathrm{Cl}$ & 18.3 & 16.0 & 4.3 & 1.0 & 0.18 & 0.115 & 0.216 \\
$\mathrm{CH}$ & 18.0 & 17.1 & 2.2 & -0.7 & 0.39 & -0.31 & 0.363 \\
$\mathrm{OMe}$ & 15.7 & 14.8 & -3.0 & -6.3 & 0.42 & -0.78 & 0.634 \\
$\mathrm{CN}$ & 16.7 & 14.8 & 6.4 & 4.1 & 0.71 & 0.66 & 0.512 \\
$\mathrm{NO}_{2}$ & 16.3 & 14.4 & 7.7 & 4.9 & 0.76 & 0.79 & 0.523 \\
$\mathrm{NMe}_{2}$ & 12.2 & 11.6 & -10.7 & -13.3 & 0.61 & -1.74 & 1.132 \\
\hline
\end{tabular}


Table S4: NBO analysis of charge and spin on the substrate at the transition states for addition of Compound I to substituted benzenes.

\begin{tabular}{ccccc}
\hline substituent & spin $(\mathrm{BSI})$ & charge(BSI) & spin(BSII) & charge(BSII) \\
$\mathrm{H}$ & -0.40 & +0.28 & -0.42 & +0.30 \\
$\mathrm{CN}$ & -0.40 & +0.20 & -0.36 & +0.20 \\
$\mathrm{NO}_{2}$ & -0.38 & +0.14 & -0.36 & +0.13 \\
$\mathrm{NMe}_{2}$ & -0.44 & +0.45 & -0.49 & +0.48 \\
\hline
\end{tabular}

Table S5: B3LYP activation energies (in $\mathrm{kcal} / \mathrm{mol}$ ) for addition of Compound I to substituted benzenes calculated in the gas phase and using the polarizable continuum-Poisson method as incorporated in Jaguar (see Marten, B.; Kim, K.; Cortis, C.; Friesner, R. A.; Murphy, R. B.; Ringnalda, M. N.; Sitkoff, D.; Honig, B. J. Phys. Chem. 1996, 100, 11775-11788) to represent en gros the effect of the protein matrix (the electrostatic continuum was assigned a value of $=4.0$, and a probe radius of $2.60 \AA$ ).

\begin{tabular}{ccc}
\hline substituent & $\mathrm{E}_{\mathrm{a}}(\mathrm{BSI})$ gas phase & $\mathrm{E}_{\mathrm{a}}(\mathrm{BSI})$ continuum \\
$\mathrm{H}$ & 19.1 & 24.6 \\
$\mathrm{NMe}_{2}$ & 12.2 & 16.4 \\
$\mathrm{CN}$ & 16.7 & 21.7 \\
\hline
\end{tabular}


Figure S1. UB3LYP/BS II activation energies for para addition to monosubstituted benzenes, plotted against the cationic Hammett parameter ${ }^{+}$.

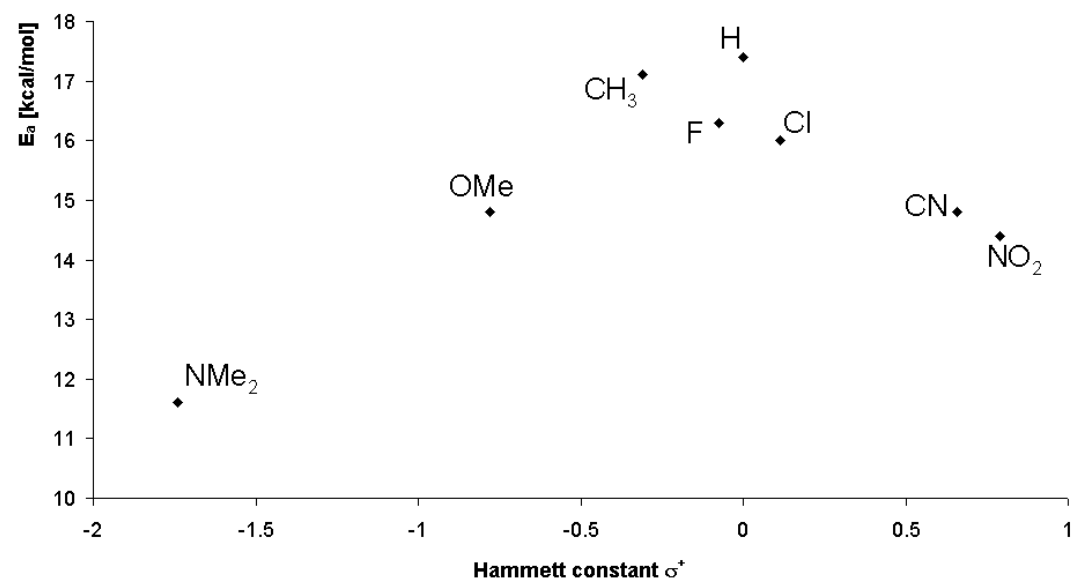

Figure S2. UB3LYP/BS II activation energies for para addition to monosubstituted benzenes, versus the radical Hammett parameter rad.

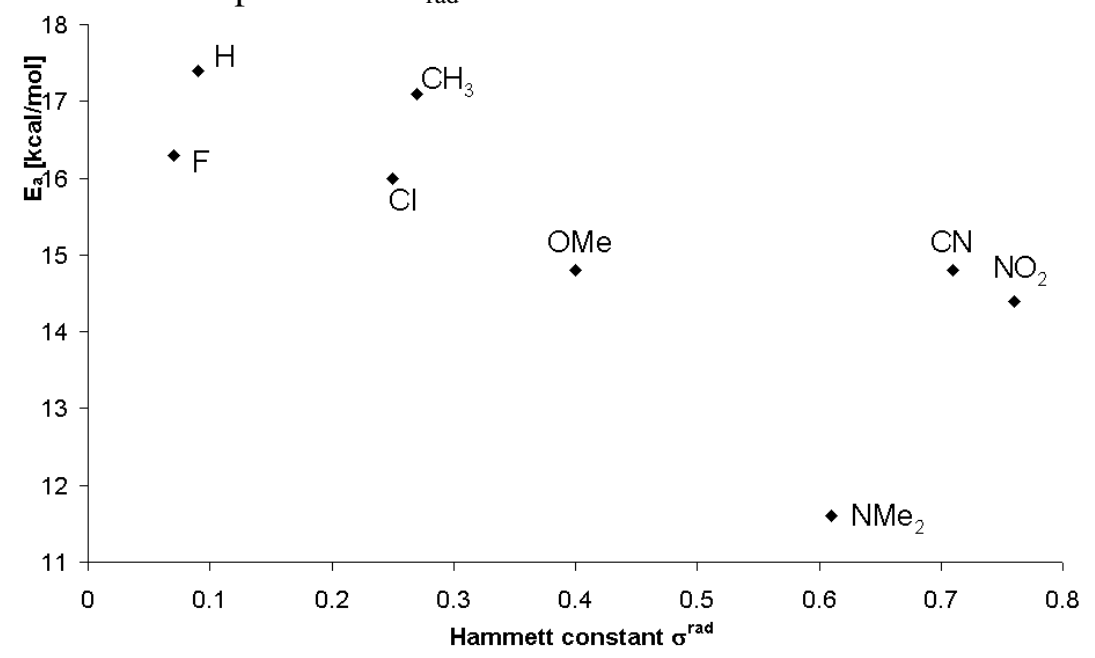


Geometries and Energies for reactants, transition states and products:

\begin{tabular}{|c|c|c|c|}
\hline \multicolumn{4}{|c|}{$\begin{array}{l}\text { Compound I } 2 \mathrm{~A} 2 \mathrm{u} \text { state } \\
\text { Etot=-1625.128503 } \\
\text { nbasis }=443 \\
\text { single point: } \\
\text { Etot }=-1625.447073 \\
\text { nbasis }=723\end{array}$} \\
\hline $\mathrm{Fe}$ & -0.4358 & -0.0521 & 0.0000 \\
\hline 0 & -2.0593 & -0.0397 & 0.0000 \\
\hline C & -0.2324 & 3.3630 & 0.0000 \\
\hline $\mathrm{N}$ & -0.2456 & -1.4809 & 1.4216 \\
\hline C & -0.1880 & -1.2932 & 2.7788 \\
\hline C & -0.2294 & -2.8397 & 1.2337 \\
\hline C & -0.1350 & -2.5633 & 3.4641 \\
\hline $\mathrm{H}$ & -0.0752 & -2.6812 & 4.5384 \\
\hline C & -0.1625 & -3.5255 & 2.5026 \\
\hline $\mathrm{H}$ & -0.1293 & -4.6011 & 2.6202 \\
\hline $\mathrm{N}$ & -0.2228 & 1.3639 & 1.4183 \\
\hline C & -0.1756 & 1.1730 & 2.7790 \\
\hline C & -0.2192 & 2.7253 & 1.2334 \\
\hline C & -0.1590 & 3.4071 & 2.5043 \\
\hline $\mathrm{H}$ & -0.1324 & 4.4826 & 2.6250 \\
\hline C & -0.1290 & 2.4424 & 3.4640 \\
\hline $\mathrm{H}$ & -0.0739 & 2.5591 & 4.5388 \\
\hline $\mathrm{N}$ & -0.2228 & 1.3639 & -1.4183 \\
\hline C & -0.2192 & 2.7253 & -1.2334 \\
\hline C & -0.1756 & 1.1730 & -2.7790 \\
\hline C & -0.1590 & 3.4071 & -2.5043 \\
\hline $\mathrm{H}$ & -0.1324 & 4.4826 & -2.6250 \\
\hline C & -0.1290 & 2.4424 & -3.4640 \\
\hline $\mathrm{H}$ & -0.0739 & 2.5591 & -4.5388 \\
\hline $\mathrm{N}$ & -0.2456 & -1.4809 & -1.4216 \\
\hline C & -0.2294 & -2.8397 & -1.2337 \\
\hline C & -0.1880 & -1.2932 & -2.7788 \\
\hline C & -0.1350 & -2.5633 & -3.4641 \\
\hline $\mathrm{H}$ & -0.0752 & -2.6812 & -4.5384 \\
\hline C & -0.1625 & -3.5255 & -2.5026 \\
\hline $\mathrm{H}$ & -0.1293 & -4.6011 & -2.6202 \\
\hline C & -0.1648 & -0.0596 & 3.4154 \\
\hline C & -0.2346 & -3.4769 & 0.0000 \\
\hline C & -0.1648 & -0.0596 & -3.4154 \\
\hline $\mathrm{H}$ & -0.1185 & -0.0593 & 4.5004 \\
\hline $\mathrm{H}$ & -0.2116 & -4.5627 & 0.0000 \\
\hline $\mathrm{H}$ & -0.1185 & -0.0593 & -4.5004 \\
\hline $\mathrm{H}$ & -0.2232 & 4.4491 & 0.0000 \\
\hline$S$ & 2.1930 & -0.0901 & 0.0000 \\
\hline C & 2.9309 & 1.5749 & 0.0000 \\
\hline $\mathrm{H}$ & 2.6608 & 2.1437 & -0.8946 \\
\hline $\mathrm{H}$ & 2.6608 & 2.1437 & 0.8946 \\
\hline 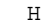 & 4.0189 & 1.4337 & 0.0000 \\
\hline
\end{tabular}

benzene adduct

Etot $=-1857.365086$

nbasis $=539$

single point

Etot $=-1857.755301$

nbasis $=891$

$\begin{array}{rrrr}\text { Fe } & 0.2600 & 0.3241 & 0.0000 \\ \mathrm{O} & -1.6735 & 0.5428 & 0.0000 \\ \mathrm{C} & 0.2497 & 3.7517 & 0.0000 \\ \mathrm{~N} & 0.2301 & -1.1416 & 1.4101 \\ \mathrm{C} & 0.2826 & -0.9309 & 2.7738 \\ \mathrm{C} & 0.4172 & -2.4926 & 1.2319 \\ \mathrm{C} & 0.4343 & -2.1893 & 3.4651 \\ \mathrm{H} & 0.4939 & -2.2981 & 4.5409 \\ \mathrm{C} & 0.5275 & -3.1558 & 2.5081 \\ \mathrm{H} & 0.6737 & -4.2211 & 2.6382 \\ \mathrm{~N} & 0.2703 & 1.7537 & 1.4152 \\ \mathrm{C} & 0.2580 & 1.5492 & 2.7661 \\ \mathrm{C} & 0.2559 & 3.1116 & 1.2340 \\ \mathrm{C} & 0.2453 & 3.7887 & 2.5126 \\ \mathrm{H} & 0.2397 & 4.8636 & 2.6422 \\ \mathrm{C} & 0.2463 & 2.8170 & 3.4660 \\ \mathrm{H} & 0.2421 & 2.9269 & 4.5433 \\ \mathrm{~N} & 0.2703 & 1.7537 & -1.4152 \\ \mathrm{C} & 0.2559 & 3.1116 & -1.2340 \\ \mathrm{C} & 0.2580 & 1.5492 & -2.7661 \\ \mathrm{C} & 0.2453 & 3.7887 & -2.5126 \\ \mathrm{H} & 0.2397 & 4.8636 & -2.6422 \\ \mathrm{C} & 0.2463 & 2.8170 & -3.4660 \\ \mathrm{H} & 0.2421 & 2.9269 & -4.5433 \\ \mathrm{~N} & 0.2301 & -1.1416 & -1.4101\end{array}$

$\begin{array}{lrrr}\text { C } & 0.4172 & -2.4926 & -1.2319 \\ \text { C } & 0.2826 & -0.9309 & -2.7738 \\ \text { C } & 0.4343 & -2.1893 & -3.4651 \\ \text { H } & 0.4939 & -2.2981 & -4.5409 \\ \text { C } & 0.5275 & -3.1558 & -2.5081 \\ \text { H } & 0.6737 & -4.2211 & -2.6382 \\ \text { C } & 0.2599 & 0.3068 & 3.3999 \\ \text { C } & 0.4933 & -3.1318 & 0.0000 \\ \text { C } & 0.2599 & 0.3068 & -3.3999 \\ \text { H } & 0.2848 & 0.3094 & 4.4863 \\ \text { H } & 0.6663 & -4.2042 & 0.0000 \\ \text { H } & 0.2848 & 0.3094 & -4.4863 \\ \text { H } & 0.2406 & 4.8381 & 0.0000 \\ \text { S } & 2.5512 & 0.1477 & 0.0000 \\ \text { C } & 3.2894 & 1.8228 & 0.0000 \\ \text { H } & 3.0028 & 2.3894 & -0.8907 \\ \text { H } & 3.0028 & 2.3894 & 0.8907 \\ \text { H } & 4.3772 & 1.6971 & 0.0000 \\ \text { C } & -2.4931 & -0.5353 & 0.0000 \\ \text { C } & -2.5933 & -1.3412 & 1.2518 \\ \text { C } & -2.9315 & -2.6751 & 1.2329 \\ \text { C } & -3.0629 & -3.3461 & 0.0000 \\ \text { C } & -2.9315 & -2.6751 & -1.2329 \\ \text { C } & -2.5933 & -1.3412 & -1.2518 \\ \text { H } & -2.4771 & -0.7921 & 2.1799 \\ \text { H } & -3.0285 & -3.2318 & 2.1607 \\ \text { H } & -3.0285 & -3.2318 & -2.1607 \\ \text { H } & -2.4771 & -0.7921 & -2.1799 \\ \text { H } & -3.5601 & -0.1263 & 0.0000 \\ \text { H } & -3.2924 & -4.4089 & 0.0000 \\ & & & \end{array}$

benzene

Etot $=-232.243798$

nbasis $=96$

single point

Etot $=-232.310900$

nbasis $=168$

$\begin{array}{lrrr}\text { C } & 1.3980 & 0.0000 & 0.0000 \\ \text { C } & -1.3980 & 0.0000 & 0.0000 \\ \text { C } & -0.6990 & -1.2107 & 0.0000 \\ \text { C } & 0.6990 & 1.2107 & 0.0000 \\ \text { C } & -0.6990 & 1.2107 & 0.0000 \\ \text { C } & 0.6990 & -1.2107 & 0.0000 \\ \text { H } & 2.4848 & 0.0000 & 0.0000 \\ \text { H } & 1.2424 & 2.1519 & 0.0000 \\ \text { H } & -1.2424 & 2.1519 & 0.0000 \\ \text { H } & -2.4848 & 0.0000 & 0.0000 \\ \text { H } & -1.2424 & -2.1519 & 0.0000 \\ \text { H } & 1.2424 & -2.1519 & 0.0000\end{array}$

benzene-Compound transition state

Etot $=-1857.341866$

nbasis $=539$

single point

Etot $=-1857.730277$

nbasis $=891$

$\begin{array}{rrrr}\mathrm{Fe} & 0.2154 & 0.2728 & 0.0000 \\ \mathrm{O} & -1.4977 & 0.3586 & 0.0000 \\ \mathrm{C} & 0.1566 & 3.7119 & 0.0000 \\ \mathrm{~N} & 0.3948 & -1.1413 & 1.4171 \\ \mathrm{C} & 0.4330 & -0.9381 & 2.7771 \\ \mathrm{C} & 0.5131 & -2.4940 & 1.2302 \\ \mathrm{C} & 0.5603 & -2.2020 & 3.4628 \\ \mathrm{H} & 0.6151 & -2.3132 & 4.5383 \\ \mathrm{C} & 0.6126 & -3.1667 & 2.5034 \\ \mathrm{H} & 0.7186 & -4.2373 & 2.6254 \\ \mathrm{~N} & 0.2787 & 1.7166 & 1.4243 \\ \mathrm{C} & 0.3132 & 1.5236 & 2.7804 \\ \mathrm{C} & 0.2002 & 3.0732 & 1.2318 \\ \mathrm{C} & 0.1914 & 3.7556 & 2.5049 \\ \mathrm{H} & 0.1421 & 4.8305 & 2.6256 \\ \mathrm{C} & 0.2636 & 2.7939 & 3.4663 \\ \mathrm{H} & 0.2853 & 2.9130 & 4.5422 \\ \mathrm{~N} & 0.2787 & 1.7166 & -1.4243 \\ \mathrm{C} & 0.2002 & 3.0732 & -1.2318 \\ \mathrm{C} & 0.3132 & 1.5236 & -2.7804 \\ \mathrm{C} & 0.1914 & 3.7556 & -2.5049 \\ \mathrm{H} & 0.1421 & 4.8305 & -2.6256 \\ \mathrm{C} & 0.2636 & 2.7939 & -3.4663 \\ \mathrm{H} & 0.2853 & 2.9130 & -4.5422 \\ \mathrm{~N} & 0.3948 & -1.1413 & -1.4171 \\ \mathrm{C} & 0.5131 & -2.4940 & -1.2302\end{array}$

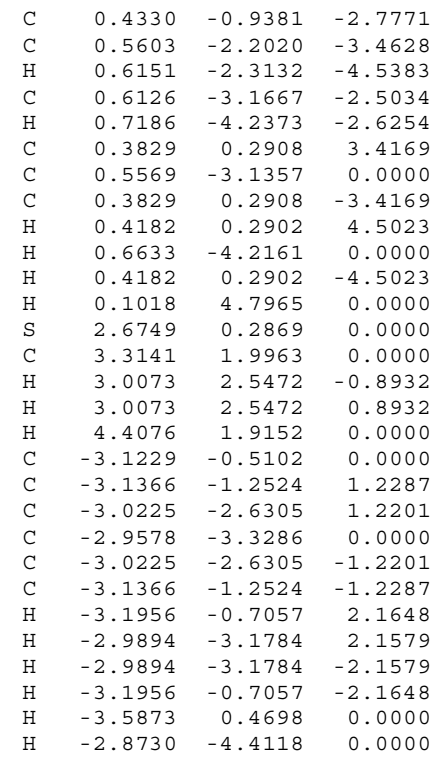

fluorobenzene adduct

Etot $=-1956.601936$

nbasis $=551$

single point

Etot $=-1957.026903$

nbasis $=907$

$\begin{array}{rrrr}\text { Fe } & 0.3732 & 0.4791 & 0.0000 \\ \mathrm{O} & -1.5542 & 0.7058 & 0.0000 \\ \mathrm{C} & 0.3693 & 3.9077 & 0.0000 \\ \mathrm{~N} & 0.3434 & -0.9860 & 1.4105 \\ \mathrm{C} & 0.3878 & -0.7749 & 2.7749 \\ \mathrm{C} & 0.5177 & -2.3379 & 1.2329 \\ \mathrm{C} & 0.5258 & -2.0350 & 3.4665 \\ \mathrm{H} & 0.5780 & -2.1443 & 4.5427 \\ \mathrm{C} & 0.6168 & -3.0024 & 2.5101 \\ \mathrm{H} & 0.7546 & -4.0686 & 2.6410 \\ \mathrm{~N} & 0.3880 & 1.9099 & 1.4160 \\ \mathrm{C} & 0.3709 & 1.7059 & 2.7669 \\ \mathrm{C} & 0.3747 & 3.2678 & 1.2341 \\ \mathrm{C} & 0.3626 & 3.9453 & 2.5125 \\ \mathrm{H} & 0.3574 & 5.0203 & 2.6417 \\ \mathrm{C} & 0.3598 & 2.9742 & 3.4664 \\ \mathrm{H} & 0.3535 & 3.0848 & 4.5435 \\ \mathrm{~N} & 0.3880 & 1.9099 & -1.4160 \\ \mathrm{C} & 0.3747 & 3.2678 & -1.2341 \\ \mathrm{C} & 0.3709 & 1.7059 & -2.7669 \\ \mathrm{C} & 0.3626 & 3.9453 & -2.5125 \\ \mathrm{H} & 0.3574 & 5.0203 & -2.6417 \\ \mathrm{C} & 0.3598 & 2.9742 & -3.4664 \\ \mathrm{H} & 0.3535 & 3.0848 & -4.5435 \\ \mathrm{~N} & 0.3434 & -0.9860 & -1.4105 \\ \mathrm{C} & 0.5177 & -2.3379 & -1.2329 \\ \mathrm{C} & 0.3878 & -0.7749 & -2.7749 \\ \mathrm{C} & 0.5258 & -2.0350 & -3.4665 \\ \mathrm{H} & 0.5780 & -2.1443 & -4.5427 \\ \mathrm{C} & 0.6168 & -3.0024 & -2.5101 \\ \mathrm{H} & 0.7546 & -4.0686 & -2.6410 \\ \mathrm{C} & 0.3673 & 0.4633 & 3.4007 \\ \mathrm{C} & 0.5904 & -2.9776 & 0.0000 \\ \mathrm{C} & 0.3673 & 0.4633 & -3.4007 \\ \mathrm{H} & 0.3895 & 0.4662 & 4.4872 \\ \mathrm{H} & 0.7526 & -4.0517 & 0.0000 \\ \mathrm{H} & 0.3895 & 0.4662 & -4.4872 \\ \mathrm{H} & 0.3609 & 4.9941 & 0.0000 \\ \mathrm{~S} & 2.6658 & 0.3104 & 0.0000 \\ \mathrm{C} & 3.4063 & 1.9837 & 0.0000 \\ \mathrm{H} & 3.1216 & 2.5514 & -0.8907 \\ \mathrm{H} & 3.1216 & 2.5514 & 0.8907 \\ \mathrm{H} & 4.4941 & 1.8563 & 0.0000 \\ \mathrm{C} & -2.3989 & -0.3563 & 0.0000 \\ \mathrm{C} & -2.4810 & -1.1710 & 1.2533 \\ \mathrm{C} & -2.7877 & -2.5072 & 1.2437 \\ \mathrm{C} & -2.8989 & -3.1540 & 0.0000 \\ & & & \\ & & & \end{array}$




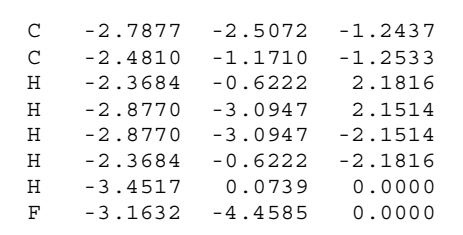

fluorobenzene

Etot $=-331.476866$

nbasis $=108$

single point

Etot $=-331.579530$

nbasis $=184$

$\begin{array}{lrrr}\text { C } & 0.0236 & 1.9095 & 0.0000 \\ \text { C } & -0.0117 & -0.8617 & 0.0000 \\ \text { C } & -0.0020 & -0.1891 & -1.2178 \\ \text { C } & 0.0152 & 1.2077 & 1.2096 \\ \text { C } & -0.0020 & -0.1891 & 1.2178 \\ \text { C } & 0.0152 & 1.2077 & -1.2096 \\ \text { H } & 0.0372 & 2.9955 & 0.0000 \\ \text { H } & 0.0224 & 1.7463 & 2.1533 \\ \text { H } & -0.0085 & -0.7559 & 2.1434 \\ \text { H } & -0.0085 & -0.7559 & -2.1434 \\ \text { H } & 0.0224 & 1.7463 & -2.1533 \\ \text { F } & -0.0276 & -2.2126 & 0.0000\end{array}$

fluorobenzene-Compound I

transition state

Etot $=-1956.576685$

nbasis $=551$

single point
Etot $=-1957.000675$

Etot $=-1957$
nbasis $=907$

$\begin{array}{rrrr}\text { nbasis }=907 & & \\ \mathrm{Fe} & 0.2963 & 0.4358 & 0.0000 \\ \mathrm{O} & -1.4175 & 0.5455 & 0.0000 \\ \mathrm{C} & 0.2714 & 3.8782 & 0.0000 \\ \mathrm{~N} & 0.4624 & -0.9782 & 1.4165 \\ \mathrm{C} & 0.4994 & -0.7748 & 2.7767 \\ \mathrm{C} & 0.5734 & -2.3320 & 1.2301 \\ \mathrm{C} & 0.6167 & -2.0396 & 3.4625 \\ \mathrm{H} & 0.6694 & -2.1509 & 4.5381 \\ \mathrm{C} & 0.6650 & -3.0050 & 2.5035 \\ \mathrm{H} & 0.7635 & -4.0763 & 2.6255 \\ \mathrm{~N} & 0.3695 & 1.8813 & 1.4245 \\ \mathrm{C} & 0.4016 & 1.6880 & 2.7804 \\ \mathrm{C} & 0.3079 & 3.2388 & 1.2317 \\ \mathrm{C} & 0.3075 & 3.9217 & 2.5047 \\ \mathrm{H} & 0.2716 & 4.9972 & 2.6250 \\ \mathrm{C} & 0.3680 & 2.9591 & 3.4662 \\ \mathrm{H} & 0.3925 & 3.0780 & 4.5420 \\ \mathrm{~N} & 0.3695 & 1.8813 & -1.4245 \\ \mathrm{C} & 0.3079 & 3.2388 & -1.2317 \\ \mathrm{C} & 0.4016 & 1.6880 & -2.7804 \\ \mathrm{C} & 0.3075 & 3.9217 & -2.5047 \\ \mathrm{H} & 0.2716 & 4.9972 & -2.6250 \\ \mathrm{C} & 0.3680 & 2.9591 & -3.4662 \\ \mathrm{H} & 0.3925 & 3.0780 & -4.5420 \\ \mathrm{~N} & 0.4624 & -0.9782 & -1.4165 \\ \mathrm{C} & 0.5734 & -2.3320 & -1.2301 \\ \mathrm{C} & 0.4994 & -0.7748 & -2.7767 \\ \mathrm{C} & 0.6167 & -2.0396 & -3.4625 \\ \mathrm{H} & 0.6694 & -2.1509 & -4.5381 \\ \mathrm{C} & 0.6650 & -3.0050 & -2.5035 \\ \mathrm{H} & 0.7635 & -4.0763 & -2.6255 \\ \mathrm{C} & 0.4585 & 0.4544 & 3.4168 \\ \mathrm{C} & 0.6119 & -2.9747 & 0.0000 \\ \mathrm{C} & 0.4585 & 0.4544 & -3.4168 \\ \mathrm{H} & 0.4938 & 0.4532 & 4.5022 \\ \mathrm{H} & 0.7029 & -4.0567 & 0.0000 \\ \mathrm{H} & -2.7774 & -3.0530 & -2.1492 \\ \mathrm{H} & 0.4938 & 0.4532 & -4.5022 \\ \mathrm{H} & 0.2297 & 4.9634 & 0.0000 \\ \mathrm{~S} & 2.7474 & 0.4305 & 0.0000 \\ \mathrm{C} & 3.4056 & 2.1331 & 0.0000 \\ \mathrm{C} & -3.0212 & -1.1120 & -1.2285 \\ \mathrm{H} & 3.1049 & 2.6876 & -0.8930 \\ \mathrm{H} & 3.1049 & 2.6876 & 0.8930 \\ \mathrm{H} & 4.4981 & 2.0405 & 0.0000 \\ \mathrm{C} & -3.0317 & -0.3699 & 0.0000 \\ \mathrm{C} & -3.0212 & -1.1120 & 1.2285 \\ \mathrm{C} & -2.8451 & -2.4826 & 1.2290 \\ \mathrm{C} & -2.7460 & -3.1468 & 0.0000 \\ \mathrm{H} & -2.8451 & -2.4826 & -1.2290 \\ \mathrm{H} & -37164 & -0.5716 & 2.1658 \\ \mathrm{H} & & -2.1658\end{array}$

\section{$\mathrm{H} \quad-3.5206 \quad 0.5977$ \\ cyanobenzene adduct \\ Etot $=-1949.602850$ \\ nbasis $=565$ \\ single point \\ Etot $=-1950.017388$ \\ nbasis $=929$}

$\begin{array}{rrrr}\mathrm{Fe} & 0.4092 & 0.5673 & 0.0000 \\ \mathrm{O} & -1.5020 & 0.8314 & 0.0000 \\ \mathrm{C} & 0.4035 & 3.9912 & 0.0000 \\ \mathrm{~N} & 0.3817 & -0.8959 & 1.4081 \\ \mathrm{C} & 0.4342 & -0.6879 & 2.7747 \\ \mathrm{C} & 0.5502 & -2.2488 & 1.2323 \\ \mathrm{C} & 0.5714 & -1.9484 & 3.4646 \\ \mathrm{H} & 0.6284 & -2.0592 & 4.5402 \\ \mathrm{C} & 0.6525 & -2.9152 & 2.5076 \\ \mathrm{H} & 0.7838 & -3.9824 & 2.6357 \\ \mathrm{~N} & 0.4364 & 1.9950 & 1.4165 \\ \mathrm{C} & 0.4214 & 1.7924 & 2.7671 \\ \mathrm{C} & 0.4141 & 3.3532 & 1.2352 \\ \mathrm{C} & 0.3972 & 4.0306 & 2.5129 \\ \mathrm{H} & 0.3826 & 5.1054 & 2.6422 \\ \mathrm{C} & 0.4030 & 3.0593 & 3.4668 \\ \mathrm{H} & 0.3956 & 3.1689 & 4.5439 \\ \mathrm{~N} & 0.4364 & 1.9950 & -1.4165 \\ \mathrm{C} & 0.4141 & 3.3532 & -1.2352 \\ \mathrm{C} & 0.4214 & 1.7924 & -2.7671 \\ \mathrm{C} & 0.3972 & 4.0306 & -2.5129 \\ \mathrm{H} & 0.3826 & 5.1054 & -2.6422 \\ \mathrm{C} & 0.4030 & 3.0593 & -3.4668 \\ \mathrm{H} & 0.3956 & 3.1689 & -4.5439 \\ \mathrm{~N} & 0.3817 & -0.8959 & -1.4081 \\ \mathrm{C} & 0.5502 & -2.2488 & -1.2323 \\ \mathrm{C} & 0.4342 & -0.6879 & -2.7747 \\ \mathrm{C} & 0.5714 & -1.9484 & -3.4646 \\ \mathrm{H} & 0.6284 & -2.0592 & -4.5402 \\ \mathrm{C} & 0.6525 & -2.9152 & -2.5076 \\ \mathrm{H} & 0.7838 & -3.9824 & -2.6357 \\ \mathrm{C} & 0.4223 & 0.5485 & 3.4001 \\ \mathrm{C} & 0.6192 & -2.8891 & 0.0000 \\ \mathrm{H} & 0.4223 & 0.5485 & -3.4001 \\ \mathrm{H} & -2.3581 & -0.4827 & -2.1832 \\ \mathrm{~N} & -3.2225 & -5.6171 & 0.0000 \\ \mathrm{C} & -3.0417 & -4.4671 & 0.0000 \\ \mathrm{H} & 0.4497 & 0.5503 & 4.4864 \\ \mathrm{H} & 0.7750 & -3.9640 & 0.0000 \\ \mathrm{H} & -2.3581 & -0.4827 & 2.1832 \\ \mathrm{H} & 0.4497 & 0.5503 & -4.4864 \\ \mathrm{H} & 0.3854 & 5.0774 & 0.0000 \\ \mathrm{~S} & 2.7119 & 0.3940 & 0.0000 \\ \mathrm{C} & 3.4502 & 2.0675 & 0.0000 \\ \mathrm{H} & 3.1671 & 2.6350 & -0.8913 \\ \mathrm{H} & 3.1671 & 2.6350 & 0.8913 \\ \mathrm{H} & 4.5378 & 1.9376 & 0.0000 \\ \mathrm{C} & -2.3726 & -0.2217 & 0.0000 \\ \mathrm{C} & -2.4537 & -1.0322 & 1.2530 \\ \mathrm{C} & -2.7239 & -2.3747 & 1.2422 \\ \mathrm{C} & -2.8182 & -3.0590 & 0.0000 \\ \mathrm{H} & -2.4239 & -2.3747 & -1.2422 \\ \mathrm{H} & -2.7977 & -2.9425 & 2.1641 \\ \mathrm{H} & -2.9425 & -2.1641 \\ \mathrm{H} & -2537 & \end{array}$

cyanobenzene-Compound I

transition state

Etot $=-1949.586422$

nbasis $=565$

single point
Etot $=-1950.000330$

nbasis $=929$

$\begin{array}{rrrr}\text { Fe } & 0.3493 & 0.5177 & 0.0000 \\ \mathrm{O} & -1.3649 & 0.6117 & 0.0000 \\ \mathrm{C} & 0.2841 & 3.9467 & 0.0000 \\ \mathrm{~N} & 0.5215 & -0.9028 & 1.4158 \\ \mathrm{C} & 0.5771 & -0.7029 & 2.7761 \\ \mathrm{C} & 0.6069 & -2.2587 & 1.2300 \\ \mathrm{C} & 0.6836 & -1.9690 & 3.4607 \\ \mathrm{H} & 0.7455 & -2.0820 & 4.5356 \\ \mathrm{C} & 0.7024 & -2.9345 & 2.5017 \\ \mathrm{H} & 0.7811 & -4.0073 & 2.6209 \\ \mathrm{~N} & 0.4360 & 1.9526 & 1.4224 \\ \mathrm{C} & 0.4744 & 1.7597 & 2.7795 \\ \mathrm{C} & 0.3388 & 3.3093 & 1.2321 \\ \mathrm{C} & 0.3235 & 3.9899 & 2.5051 \\ \mathrm{H} & 0.2584 & 5.0635 & 2.6273\end{array}$

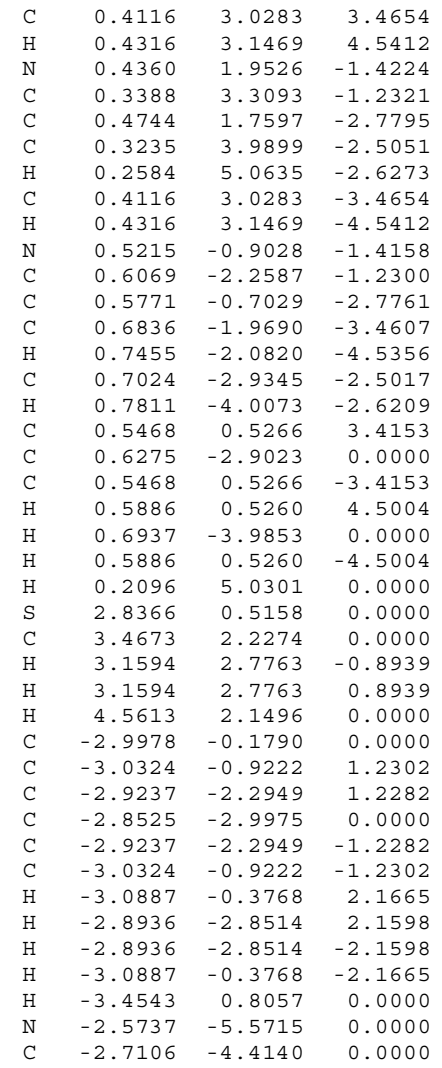

cyanobenzene

Etot $=-324.484569$

nbasis $=122$

single point

Etot $=-324.576882$

nbasis $=206$

$\begin{array}{crrr}\text { C } & 0.0246 & 2.2426 & 0.0000 \\ \text { C } & -0.0164 & -0.5485 & 0.0000 \\ \text { C } & -0.0052 & 0.1525 & -1.2180 \\ \text { C } & 0.0146 & 1.5455 & 1.2118 \\ \text { C } & -0.0052 & 0.1525 & 1.2180 \\ \text { C } & 0.0146 & 1.5455 & -1.2118 \\ \text { H } & 0.0405 & 3.3290 & 0.0000 \\ \text { H } & 0.0222 & 2.0865 & 2.1537 \\ \text { H } & -0.0118 & -0.3988 & 2.1533 \\ \text { H } & -0.0118 & -0.3988 & -2.1533 \\ \text { H } & 0.0222 & 2.0865 & -2.1537 \\ \text { N } & -0.0125 & -3.1458 & 0.0000 \\ \text { C } & -0.0176 & -1.9823 & 0.0000\end{array}$

nitrobenzene adduct

Etot $=-2061.858765$

nbasis $=579$

single point

Etot $=-2062.313076$

nbasis $=951$

$\begin{array}{rrrr}\mathrm{Fe} & 0.3937 & 0.5720 & 0.0012 \\ \mathrm{O} & -1.5148 & 0.8121 & 0.0189 \\ \mathrm{C} & 0.4207 & 3.9997 & -0.0122 \\ \mathrm{~N} & 0.3875 & -0.8794 & 1.4143 \\ \mathrm{C} & 0.4387 & -0.6687 & 2.7778 \\ \mathrm{C} & 0.5555 & -2.2340 & 1.2424 \\ \mathrm{C} & 0.5738 & -1.9264 & 3.4729 \\ \mathrm{H} & 0.6250 & -2.0333 & 4.5492 \\ \mathrm{C} & 0.6555 & -2.8965 & 2.5195 \\ \mathrm{H} & 0.7782 & -3.9640 & 2.6512 \\ \mathrm{~N} & 0.4375 & 2.0092 & 1.4109 \\ \mathrm{C} & 0.4277 & 1.8123 & 2.7640 \\ \mathrm{C} & 0.4277 & 3.3661 & 1.2265 \\ \mathrm{C} & 0.4242 & 4.0490 & 2.5008 \\ \mathrm{H} & 0.4230 & 5.1244 & 2.6256 \\ \mathrm{C} & 0.4243 & 3.0815 & 3.4588 \\ \mathrm{H} & 0.4236 & 3.1958 & 4.5354 \\ \mathrm{~N} & 0.4274 & 1.9975 & -1.4212 \\ \mathrm{C} & 0.4207 & 3.3578 & -1.2444 \\ \mathrm{C} & 0.4126 & 1.7930 & -2.7712\end{array}$




$\begin{array}{rrrr}\mathrm{C} & 0.4132 & 4.0318 & -2.5236 \\ \mathrm{H} & 0.4134 & 5.1063 & -2.6558 \\ \mathrm{C} & 0.4085 & 3.0576 & -3.4746 \\ \mathrm{H} & 0.4047 & 3.1646 & -4.5520 \\ \mathrm{~N} & 0.3755 & -0.8907 & -1.4020 \\ \mathrm{C} & 0.5458 & -2.2418 & -1.2236 \\ \mathrm{C} & 0.4193 & -0.6877 & -2.7697 \\ \mathrm{C} & 0.5505 & -1.9506 & -3.4565 \\ \mathrm{H} & 0.5966 & -2.0649 & -4.5321 \\ \mathrm{C} & 0.6386 & -2.9137 & -2.4967 \\ \mathrm{H} & 0.7629 & -3.9819 & -2.6219 \\ \mathrm{C} & 0.4264 & 0.5712 & 3.3996 \\ \mathrm{C} & 0.6234 & -2.8767 & 0.0122 \\ \mathrm{C} & 0.4078 & 0.5462 & -3.3996 \\ \mathrm{H} & 0.4537 & 0.5760 & 4.4859 \\ \mathrm{H} & 0.7808 & -3.9513 & 0.0154 \\ \mathrm{H} & 0.4327 & 0.5439 & -4.4859 \\ \mathrm{H} & 0.4160 & 5.0861 & -0.0162 \\ \mathrm{~S} & 2.7126 & 0.4090 & -0.0155 \\ \mathrm{C} & 3.4572 & 2.0789 & 0.0106 \\ \mathrm{H} & 3.1687 & 2.6664 & -0.8660 \\ \mathrm{H} & 3.1905 & 2.6294 & 0.9172 \\ \mathrm{H} & 4.5439 & 1.9411 & -0.0059 \\ \mathrm{C} & -2.3912 & -0.2428 & 0.0066 \\ \mathrm{C} & -2.4625 & -1.0809 & 1.2443 \\ \mathrm{C} & -2.7140 & -2.4278 & 1.2102 \\ \mathrm{C} & -2.8112 & -3.0680 & -0.0442 \\ \mathrm{C} & -2.7454 & -2.3782 & -1.2738 \\ \mathrm{C} & -2.4863 & -1.0329 & -1.2602 \\ \mathrm{H} & -2.3720 & -0.5501 & 2.1858 \\ \mathrm{H} & -2.7773 & -3.0266 & 2.1101 \\ \mathrm{H} & -2.8345 & -2.9398 & -2.1953 \\ \mathrm{H} & -2.4086 & -0.4672 & -2.1824 \\ \mathrm{H} & -3.4140 & 0.2429 & 0.0248 \\ \mathrm{~N} & -3.0195 & -4.5185 & -0.0719 \\ \mathrm{O} & -3.0235 & -5.1201 & 1.0094 \\ \mathrm{O} & -3.1740 & -5.0560 & -1.1755\end{array}$

\begin{tabular}{|c|c|c|c|}
\hline \multicolumn{4}{|c|}{$\begin{array}{l}\text { nitrobenzene-Compound } \\
\text { transition state } \\
\text { Etot }=-2061.844686 \\
\text { nbasis }=579 \\
\text { single point } \\
\text { Etot }=-2062.297970 \\
\text { nbasis }=951\end{array}$} \\
\hline $\mathrm{Fe}$ & 0.2511 & 0.4648 & -0.0422 \\
\hline 0 & -1.4612 & 0.5380 & -0.0589 \\
\hline C & 0.2882 & 3.8968 & -0.0359 \\
\hline $\mathrm{N}$ & 0.3998 & -0.9572 & 1.3759 \\
\hline C & 0.4322 & -0.7649 & 2.7371 \\
\hline $\mathrm{C}$ & 0.4833 & -2.3117 & 1.1860 \\
\hline C & 0.5166 & -2.0352 & 3.4188 \\
\hline $\mathrm{H}$ & 0.5540 & -2.1534 & 4.4941 \\
\hline C & 0.5474 & -2.9962 & 2.4559 \\
\hline $\mathrm{H}$ & 0.6024 & -4.0709 & 2.5705 \\
\hline $\mathrm{N}$ & 0.3658 & 1.8927 & 1.3820 \\
\hline C & 0.3801 & 1.6984 & 2.7405 \\
\hline C & 0.3148 & 3.2533 & 1.1943 \\
\hline C & 0.3086 & 3.9319 & 2.4667 \\
\hline $\mathrm{H}$ & 0.2778 & 5.0071 & 2.5894 \\
\hline C & 0.3505 & 2.9663 & 3.4272 \\
\hline $\mathrm{H}$ & 0.3615 & 3.0828 & 4.5034 \\
\hline $\mathrm{N}$ & 0.3460 & 1.9030 & -1.4630 \\
\hline C & 0.30 & 3.2617 & -1.2704 \\
\hline C & 0.3525 & 1.7113 & -2.8221 \\
\hline C & 0.2960 & 3.9450 & -2.5424 \\
\hline $\mathrm{H}$ & 0.2731 & 5.0207 & -2.6619 \\
\hline C & 0.3238 & 2.9825 & -3.5051 \\
\hline $\mathrm{H}$ & 0.3290 & 3.1020 & -4.5811 \\
\hline $\mathrm{N}$ & 0.4176 & -0.9496 & -1.4607 \\
\hline C & 0.4951 & -2.3072 & -1.2756 \\
\hline C & 0.4318 & -0.7513 & -2.8225 \\
\hline $\mathrm{C}$ & 0.5094 & -2.0186 & -3.5074 \\
\hline $\mathrm{H}$ & 0.5353 & -2.1335 & -4.5836 \\
\hline $\mathrm{C}$ & 0.5496 & -2.9841 & -2.5481 \\
\hline $\mathrm{H}$ & 0.6049 & -4.0582 & -2.6683 \\
\hline C & 0.4151 & 0.4636 & 3.3775 \\
\hline C & 0.5152 & -2.9514 & -0.0460 \\
\hline C & 0.3889 & 0.4791 & -3.4602 \\
\hline $\mathrm{H}$ & 0.4410 & 0.4624 & 4.4630 \\
\hline $\mathrm{H}$ & 0.5793 & -4.0350 & -0.0486 \\
\hline $\mathrm{H}$ & 0.4037 & 0.4788 & -4.5459 \\
\hline $\mathrm{H}$ & 0.2556 & 4.9823 & -0.0319 \\
\hline$S$ & 2.7330 & 0.4247 & -0.2361 \\
\hline C & 3.4175 & 2.0199 & 0.3230 \\
\hline $\mathrm{H}$ & 3.0494 & 2.8475 & -0.2905 \\
\hline $\mathrm{H}$ & 3.1985 & 2.2182 & 1.375 \\
\hline$H$ & 4.5040 & 1.955 & \\
\hline
\end{tabular}

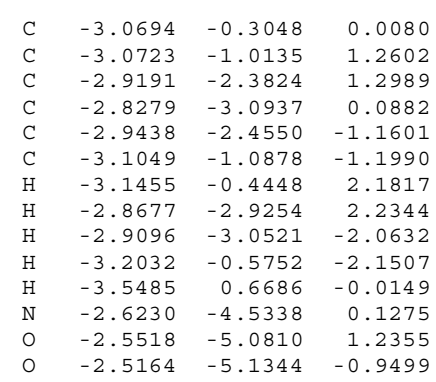

nitrobenzene

Etot $=-436.741866$

nbasis $=136$

single point

Etot $=-436.873778$

nbasis $=228$

$\begin{array}{lrrr}\text { C } & 0.0249 & 2.2487 & -0.0002 \\ \text { C } & -0.0168 & -0.5112 & 0.0012 \\ \text { C } & -0.0220 & 0.1591 & -1.2210 \\ \text { C } & 0.0277 & 1.5535 & 1.2139 \\ \text { C } & 0.0088 & 0.1598 & 1.2228 \\ \text { C } & 0.0010 & 1.5527 & -1.2135 \\ \text { H } & 0.0413 & 3.3351 & -0.0007 \\ \text { H } & 0.0452 & 2.0963 & 2.1543 \\ \text { H } & 0.0130 & -0.4082 & 2.1453 \\ \text { H } & -0.0432 & -0.4095 & -2.1430 \\ \text { H } & -0.0000 & 2.0948 & -2.1545 \\ \mathrm{~N} & -0.0385 & -1.9858 & 0.0018 \\ \mathrm{O} & 0.0009 & -2.5580 & 1.0909 \\ \mathrm{O} & -0.0947 & -2.5573 & -1.0869\end{array}$

chlorobenzene adduct

Etot $=-2316.958525$

nbasis $=555$

single point

Etot $=-2317.379136$

nbasis $=915$

$\begin{array}{rrrr}\text { Fe } & 0.4709 & 0.6423 & 0.0000 \\ \mathrm{O} & -1.4608 & 0.8622 & 0.0000 \\ \mathrm{C} & 0.4783 & 4.0707 & 0.0000 \\ \mathrm{~N} & 0.4436 & -0.8218 & 1.4091 \\ \mathrm{C} & 0.4881 & -0.6113 & 2.7740 \\ \mathrm{C} & 0.6252 & -2.1734 & 1.2321 \\ \mathrm{C} & 0.6303 & -1.8706 & 3.4652 \\ \mathrm{H} & 0.6822 & -1.9802 & 4.5413 \\ \mathrm{C} & 0.7252 & -2.8374 & 2.5086 \\ \mathrm{H} & 0.8651 & -3.9033 & 2.6392 \\ \mathrm{~N} & 0.4880 & 2.0725 & 1.4153 \\ \mathrm{C} & 0.4705 & 1.8688 & 2.7662 \\ \mathrm{C} & 0.4803 & 3.4307 & 1.2340 \\ \mathrm{C} & 0.4709 & 4.1080 & 2.5125 \\ \mathrm{H} & 0.4704 & 5.1830 & 2.6419 \\ \mathrm{C} & 0.4635 & 3.1366 & 3.4660 \\ \mathrm{H} & 0.4573 & 3.2465 & 4.5432 \\ \mathrm{~N} & 0.4880 & 2.0725 & -1.4153 \\ \mathrm{C} & 0.4803 & 3.4307 & -1.2340 \\ \mathrm{C} & 0.4705 & 1.8688 & -2.7662 \\ \mathrm{C} & 0.4709 & 4.1080 & -2.5125 \\ \mathrm{H} & 0.4704 & 5.1830 & -2.6419 \\ \mathrm{C} & 0.4635 & 3.1366 & -3.4660 \\ \mathrm{H} & 0.4573 & 3.2465 & -4.5432 \\ \mathrm{~N} & 0.4436 & -0.8218 & -1.4091 \\ \mathrm{C} & 0.6252 & -2.1734 & -1.2321 \\ \mathrm{C} & 0.4881 & -0.6113 & -2.7740 \\ \mathrm{C} & 0.6303 & -1.8706 & -3.4652 \\ \mathrm{H} & 0.6822 & -1.9802 & -4.5413 \\ \mathrm{C} & 0.7252 & -2.8374 & -2.5086 \\ \mathrm{H} & 0.8651 & -3.9033 & -2.6392 \\ \mathrm{C} & 0.4659 & 0.6262 & 3.3998 \\ \mathrm{C} & 0.7026 & -2.8125 & 0.0000 \\ \mathrm{C} & 0.4659 & 0.6262 & -3.3998 \\ \mathrm{H} & 0.4882 & 0.6288 & 4.4862 \\ \mathrm{H} & 0.8670 & -3.8860 & 0.0000 \\ \mathrm{H} & 0.4882 & 0.6288 & -4.4862 \\ \mathrm{H} & 0.4752 & 5.1571 & 0.0000 \\ \mathrm{~S} & 2.7644 & 0.4684 & 0.0000 \\ \mathrm{C} & 3.5108 & 2.1384 & 0.0000 \\ \mathrm{H} & 3.2290 & 2.7072 & -0.8909 \\ \mathrm{H} & 3.2290 & 2.7072 & 0.8909 \\ \mathrm{H} & 4.5980 & 2.0053 & 0.0000 \\ \mathrm{C} & -2.2951 & -0.2084 & 0.0000 \\ \mathrm{C} & -2.3806 & -1.0237 & 1.2503 \\ \mathrm{C} & -2.6932 & -2.3608 & 1.2411\end{array}$

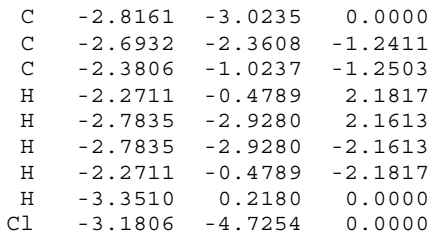

chlorobenzene-Compound I

transition state

Etot $=-2316.936235$

nbasis $=555$

single point

Etot $=-2317.355252$

nbasis $=915$

\begin{tabular}{|c|c|c|c|}
\hline $\mathrm{Fe}$ & 0.3884 & 0.6051 & 0.0000 \\
\hline 0 & -1.3298 & 0.6941 & 0.0000 \\
\hline C & 0.3808 & 4.0529 & 0.0000 \\
\hline $\mathrm{N}$ & 0.5542 & -0.8025 & 1.4163 \\
\hline $\mathrm{C}$ & 0.5754 & -0.6004 & 2.7768 \\
\hline C & 0.6718 & -2.1562 & 1.2299 \\
\hline C & 0.6909 & -1.8651 & 3.4628 \\
\hline $\mathrm{H}$ & 0.7309 & -1.9770 & 4.5389 \\
\hline C & 0.7545 & -2.8294 & 2.5036 \\
\hline $\mathrm{H}$ & 0.8528 & -3.9006 & 2.6255 \\
\hline $\mathrm{N}$ & 0.4599 & 2.0553 & 1.4243 \\
\hline C & 0.4755 & 1.8623 & 2.7809 \\
\hline C & 0.4085 & 3.4132 & 1.2319 \\
\hline C & 0.4003 & 4.0962 & 2.5050 \\
\hline $\mathrm{H}$ & 0.3708 & 5.1720 & 2.6251 \\
\hline C & 0.4416 & 3.1332 & 3.4668 \\
\hline $\mathrm{H}$ & 0.4538 & 3.2520 & 4.5428 \\
\hline N & 0.4599 & 2.0553 & -1.4243 \\
\hline C & 0.4085 & 3.4132 & -1.2319 \\
\hline C & 0.4755 & 1.8623 & -2.7809 \\
\hline C & 0.4003 & 4.0962 & -2.5050 \\
\hline $\mathrm{H}$ & 0.3708 & 5.1720 & -2.6251 \\
\hline C & 0.4416 & 3.1332 & -3.4668 \\
\hline $\mathrm{H}$ & 0.4538 & 3.2520 & -4.5428 \\
\hline $\mathrm{N}$ & 0.5542 & -0.8025 & -1.4163 \\
\hline C & 0.6718 & -2.1562 & -1.2299 \\
\hline C & 0.5754 & -0.6004 & -2.7768 \\
\hline C & 0.6909 & -1.8651 & -3.4628 \\
\hline $\mathrm{H}$ & 0.7309 & -1.9770 & -4.5389 \\
\hline C & 0.7545 & -2.8294 & -2.5036 \\
\hline $\mathrm{H}$ & 0.8528 & -3.9006 & -2.6255 \\
\hline C & 0.5237 & 0.6282 & 3.4169 \\
\hline C & 0.7165 & -2.7978 & 0.0000 \\
\hline C & 0.5237 & 0.6282 & -3.4169 \\
\hline $\mathrm{H}$ & 0.5471 & 0.6259 & 4.5026 \\
\hline $\mathrm{H}$ & 0.8124 & -3.8787 & 0.0000 \\
\hline $\mathrm{H}$ & 0.5471 & 0.6259 & -4.5026 \\
\hline $\mathrm{H}$ & 0.3469 & 5.1383 & 0.0000 \\
\hline $\mathrm{S}$ & 2.8456 & 0.6076 & 0.0000 \\
\hline C & 3.5058 & 2.3085 & 0.0000 \\
\hline $\mathrm{H}$ & 3.2063 & 2.8635 & -0.8933 \\
\hline $\mathrm{H}$ & 3.2063 & 2.8635 & 0.8933 \\
\hline $\mathrm{H}$ & 4.5982 & 2.2124 & 0.0000 \\
\hline C & -2.9233 & -0.2456 & 0.0000 \\
\hline C & -2.9261 & -0.9918 & 1.2266 \\
\hline C & -2.8042 & -2.3693 & 1.2270 \\
\hline C & -2.7406 & -3.0526 & 0.0000 \\
\hline C & -2.8042 & -2.3693 & -1.2270 \\
\hline C & -2.9261 & -0.9918 & -1.2266 \\
\hline $\mathrm{H}$ & -2.9939 & -0.4520 & 2.1650 \\
\hline $\mathrm{H}$ & -2.7708 & -2.9274 & 2.1569 \\
\hline $\mathrm{H}$ & -2.7708 & -2.9274 & -2.1569 \\
\hline $\mathrm{H}$ & -2.9939 & -0.4520 & -2.1650 \\
\hline 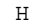 & -3.4167 & 0.7199 & 0.0000 \\
\hline $\mathrm{Cl}$ & -2.6124 & -4.8021 & 0.0000 \\
\hline
\end{tabular}

chlorobenzene

Etot $=-691.836814$

nbasis $=112$

single point

Etot $=-691.933736$

nbasis $=192$

$\begin{array}{lrrr}\text { C } & 0.0291 & 2.3560 & 0.0000 \\ \text { C } & -0.0057 & -0.4240 & 0.0000 \\ \text { C } & 0.0032 & 0.2589 & -1.2167 \\ \text { C } & 0.0205 & 1.6552 & 1.2085 \\ \text { C } & 0.0032 & 0.2589 & 1.2167 \\ \text { C } & 0.0205 & 1.6552 & -1.2085 \\ \text { H } & 0.0428 & 3.4422 & 0.0000 \\ \text { H } & 0.0274 & 2.1936 & 2.1523 \\ H & -0.0037 & -0.2950 & 2.1496\end{array}$




\begin{tabular}{|c|c|c|c|}
\hline $\mathrm{H}$ & -0.0037 & -0.2950 & -2.1496 \\
\hline $\mathrm{H}$ & 0.0274 & 2.1936 & -2.1523 \\
\hline $\mathrm{Cl}$ & -0.0269 & -2.1853 & 0.0000 \\
\hline \\
\hline \multicolumn{4}{|c|}{ Etot $=-1896.685809$} \\
\hline \multicolumn{4}{|c|}{ nbasis $=557$} \\
\hline \multicolumn{4}{|c|}{ single point } \\
\hline \multirow{2}{*}{\multicolumn{4}{|c|}{ Etot $=-1897.086504$}} \\
\hline & & & \\
\hline $\mathrm{Fe}$ & 0.3118 & 0.4844 & 0.0000 \\
\hline 0 & -1.6298 & 0.5346 & 0.0000 \\
\hline C & -0.0013 & 3.8986 & 0.0000 \\
\hline $\mathrm{N}$ & 0.4180 & -0.9763 & 1.4103 \\
\hline C & 0.4430 & -0.7625 & 2.7738 \\
\hline C & 0.7208 & -2.3054 & 1.2323 \\
\hline $\mathrm{C}$ & 0.7030 & -2.0031 & 3.4657 \\
\hline $\mathrm{H}$ & 0.7675 & -2.1066 & 4.5418 \\
\hline C & 0.8855 & -2.9572 & 2.5090 \\
\hline $\mathrm{H}$ & 1.1257 & -4.0053 & 2.6400 \\
\hline $\mathrm{N}$ & 0.1961 & 1.9103 & 1.4157 \\
\hline C & 0.1952 & 1.7049 & 2.7666 \\
\hline C & 0.0609 & 3.2613 & 1.2339 \\
\hline $\mathrm{C}$ & -0.0145 & 3.9346 & 2.5126 \\
\hline $\mathrm{H}$ & -0.1170 & 5.0047 & 2.6419 \\
\hline C & 0.0683 & 2.9668 & 3.4663 \\
\hline $\mathrm{H}$ & 0.0499 & 3.0756 & 4.5435 \\
\hline $\mathrm{N}$ & 0.1961 & 1.9103 & -1.4157 \\
\hline C & 0.0609 & 3.2613 & -1.2339 \\
\hline C & 0.1952 & 1.7049 & -2.7666 \\
\hline C & -0.0145 & 3.9346 & -2.5126 \\
\hline $\mathrm{H}$ & -0.1170 & 5.0047 & -2.6419 \\
\hline C & 0.0683 & 2.9668 & -3.4663 \\
\hline $\mathrm{H}$ & 0.0499 & 3.0756 & -4.5435 \\
\hline $\mathrm{N}$ & 0.4180 & -0.9763 & -1.4103 \\
\hline C & 0.7208 & -2.3054 & -1.2323 \\
\hline C & 0.4430 & -0.7625 & -2.7738 \\
\hline C & 0.7030 & -2.0031 & -3.4657 \\
\hline $\mathrm{H}$ & 0.7675 & -2.1066 & -4.5418 \\
\hline C & 0.8855 & -2.9572 & -2.5090 \\
\hline $\mathrm{H}$ & 1.1257 & -4.0053 & -2.6400 \\
\hline C & 0.3052 & 0.4678 & 3.4004 \\
\hline C & 0.8565 & -2.9343 & 0.0000 \\
\hline C & 0.3052 & 0.4678 & -3.4004 \\
\hline $\mathrm{H}$ & 0.3263 & 0.4721 & 4.4869 \\
\hline $\mathrm{H}$ & 1.1232 & -3.9876 & 0.0000 \\
\hline $\mathrm{H}$ & 0.32 & 0.4 & -4.4869 \\
\hline $\mathrm{H}$ & -0.1074 & 4.9798 & 0.0000 \\
\hline$S$ & 2.6109 & 0.5198 & 0.0000 \\
\hline C & 3.1965 & 2.2533 & 0.0000 \\
\hline $\mathrm{H}$ & 2.8608 & 2.7927 & -0.8906 \\
\hline $\mathrm{H}$ & 2.8608 & 2.7927 & 0.8906 \\
\hline $\mathrm{H}$ & 4.2915 & 2.2259 & 0.0000 \\
\hline $\mathrm{C}$ & -2.3700 & -0.6015 & 0.0000 \\
\hline C & -2.4007 & -1.4178 & 1.2481 \\
\hline $\mathrm{C}$ & -2.6236 & -2.7713 & 1.2280 \\
\hline C & -2.7004 & -3.4735 & 0.0000 \\
\hline C & -2.6236 & -2.7713 & -1.2280 \\
\hline C & -2.4007 & -1.4178 & -1.2481 \\
\hline $\mathrm{H}$ & -2.3267 & -0.8649 & 2.1785 \\
\hline $\mathrm{H}$ & -2.6784 & -3.3316 & 2.1581 \\
\hline $\mathrm{H}$ & -2.6784 & -3.3316 & -2.1581 \\
\hline $\mathrm{H}$ & -2.3267 & -0.8649 & -2.1785 \\
\hline $\mathrm{H}$ & -3.4623 & -0.2722 & 0.0000 \\
\hline C & -2.8649 & -4.9659 & 0.0000 \\
\hline $\mathrm{H}$ & -3.3959 & -5.3146 & -0.8909 \\
\hline $\mathrm{H}$ & -1.8772 & -5.4470 & 0.0000 \\
\hline $\mathrm{H}$ & -3.3959 & -5.3146 & 0.8909 \\
\hline
\end{tabular}

toluene-Compound I transition

\section{state}

Etot $=-1896.660411$

nbasis $=557$

single point

Etot $=-1897.058243$

nbasis $=925$

$\begin{array}{rrrr}\mathrm{Fe} & 0.2684 & 0.4230 & 0.0000 \\ \mathrm{O} & -1.4441 & 0.4027 & 0.0000 \\ \mathrm{C} & -0.0462 & 3.8506 & 0.0000 \\ \mathrm{~N} & 0.5505 & -0.9656 & 1.4230 \\ \mathrm{C} & 0.5710 & -0.7620 & 2.7823 \\ \mathrm{C} & 0.7763 & -2.3046 & 1.2326 \\ \mathrm{C} & 0.7963 & -2.0138 & 3.4656 \\ \mathrm{H} & 0.8597 & -2.1226 & 4.5410 \\ \mathrm{C} & 0.9270 & -2.9697 & 2.5047 \\ \mathrm{H} & 1.1185 & -4.0286 & 2.6256 \\ \mathrm{~N} & 0.2274 & 1.8702 & 1.4248 \\ \mathrm{C} & 0.2672 & 1.6818 & 2.7816\end{array}$

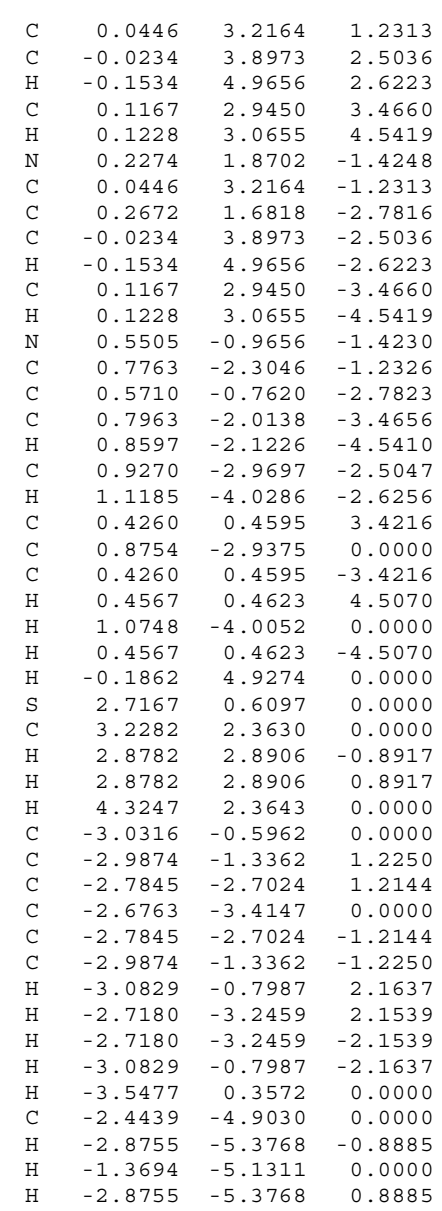

toluene adduct

Etot $=-271.560754$

nbasis $=114$

single point

Etot $=-271.638377$

nbasis $=202$

$\begin{array}{crrr}\text { nbasis }=202 \\ \text { C } & 0.0293 & 2.3668 & -0.0011 \\ \text { C } & -0.0058 & -0.4496 & 0.0040 \\ \text { C } & 0.0032 & 0.2664 & -1.2025 \\ \text { C } & 0.0206 & 1.6662 & 1.2059 \\ \text { C } & 0.0033 & 0.2692 & 1.2053 \\ \text { C } & 0.0205 & 1.6608 & -1.2077 \\ \text { H } & 0.0429 & 3.4537 & -0.0035 \\ \text { H } & 0.0274 & 2.2062 & 2.1497 \\ \text { H } & -0.0035 & -0.2714 & 2.1493 \\ \text { H } & -0.0036 & -0.2769 & -2.1455 \\ \text { H } & 0.0272 & 2.1981 & -2.1531 \\ \text { C } & -0.0242 & -1.9611 & -0.0018 \\ \text { H } & -0.0298 & -2.3637 & 1.0150 \\ \text { H } & -0.9103 & -2.3472 & -0.5190 \\ \text { H } & 0.8529 & -2.3687 & -0.5178\end{array}$

anisole adduct

Etot $=-1971.898253$

nbasis $=571$

single point

Etot $=-1972.324093$

nbasis $=947$

$\begin{array}{rrrr}\text { Fe } & 0.3571 & 0.4756 & 0.0117 \\ \mathrm{O} & -1.5683 & 0.7165 & 0.0486 \\ \mathrm{C} & 0.3709 & 3.9014 & -0.0007 \\ \mathrm{~N} & 0.3434 & -0.9847 & 1.4255 \\ \mathrm{C} & 0.3875 & -0.7719 & 2.7859 \\ \mathrm{C} & 0.4989 & -2.3378 & 1.2496 \\ \mathrm{C} & 0.5186 & -2.0315 & 3.4819 \\ \mathrm{H} & 0.5727 & -2.1381 & 4.5582 \\ \mathrm{C} & 0.5972 & -3.0018 & 2.5278 \\ \mathrm{H} & 0.7242 & -4.0693 & 2.6603 \\ \mathrm{~N} & 0.3934 & 1.9087 & 1.4219 \\ \mathrm{C} & 0.3840 & 1.7077 & 2.7738 \\ \mathrm{C} & 0.3871 & 3.2646 & 1.2356\end{array}$

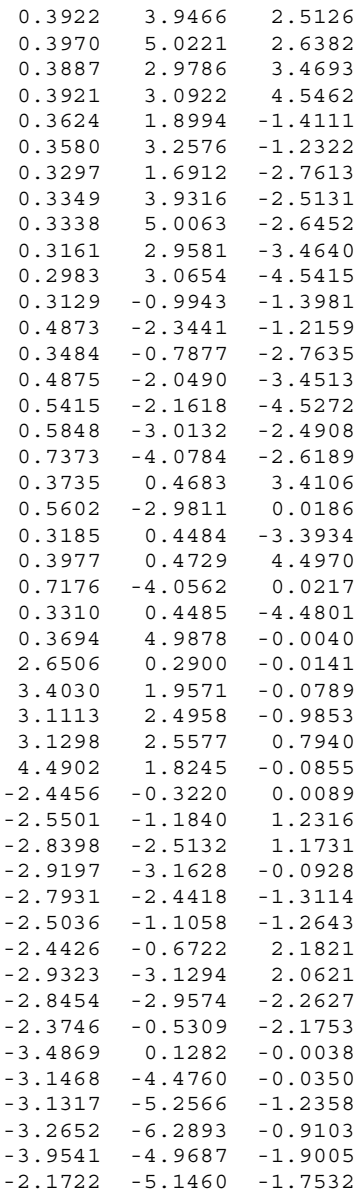

anisole-Compound I transition state

Etot $=-1971.868365$

nbasis $=571$

single point

Etot $=-1972.290503$

nbasis $=947$

$\begin{array}{rrrr}\mathrm{Fe} & 0.2591 & 0.3388 & 0.0276 \\ \mathrm{O} & -1.4432 & 0.4763 & 0.0739 \\ \mathrm{C} & 0.2460 & 3.7740 & -0.0525 \\ \mathrm{~N} & 0.4298 & -1.0472 & 1.4717 \\ \mathrm{C} & 0.4629 & -0.8152 & 2.8263 \\ \mathrm{C} & 0.5188 & -2.4067 & 1.3144 \\ \mathrm{C} & 0.5593 & -2.0665 & 3.5398 \\ \mathrm{H} & 0.6032 & -2.1557 & 4.6179 \\ \mathrm{C} & 0.5976 & -3.0534 & 2.6022 \\ \mathrm{H} & 0.6769 & -4.1232 & 2.7484 \\ \mathrm{~N} & 0.3529 & 1.8130 & 1.4180 \\ \mathrm{C} & 0.3795 & 1.6487 & 2.7778 \\ \mathrm{C} & 0.2950 & 3.1646 & 1.1947 \\ \mathrm{C} & 0.2962 & 3.8770 & 2.4531 \\ \mathrm{H} & 0.2628 & 4.9552 & 2.5490 \\ \mathrm{C} & 0.3485 & 2.9359 & 3.4354 \\ \mathrm{H} & 0.3676 & 3.0788 & 4.5085 \\ \mathrm{~N} & 0.3167 & 1.7423 & -1.4314 \\ \mathrm{C} & 0.2555 & 3.1041 & -1.2688 \\ \mathrm{C} & 0.3009 & 1.5181 & -2.7834 \\ \mathrm{C} & 0.2124 & 3.7573 & -2.5564 \\ \mathrm{H} & 0.1675 & 4.8298 & -2.7006 \\ \mathrm{C} & 0.2418 & 2.7729 & -3.4971 \\ \mathrm{H} & 0.2273 & 2.8670 & -4.5757 \\ \mathrm{~N} & 0.3739 & -1.1139 & -1.3639 \\ \mathrm{C} & 0.4870 & -2.4618 & -1.1458 \\ \mathrm{C} & 0.3838 & -0.9444 & -2.7272 \\ \mathrm{C} & 0.4889 & -2.2249 & -3.3865 \\ \mathrm{H} & 0.5245 & -2.3614 & -4.4601 \\ \mathrm{C} & 0.5589 & -3.1669 & -2.4051 \\ \mathrm{H} & 0.6702 & -4.2397 & -2.5029 \\ \mathrm{C} & 0.4271 & 0.4291 & 3.4400 \\ \mathrm{C} & 0.5432 & -3.0755 & 0.0984 \\ \mathrm{C} & 0.3337 & 0.2708 & -3.3941 \\ \mathrm{H} & & & \end{array}$




$\begin{array}{lrrr}\text { H } & 0.4552 & 0.4505 & 4.5254 \\ \mathrm{H} & 0.6275 & -4.1577 & 0.1228 \\ \mathrm{H} & 0.3365 & 0.2457 & -4.4799 \\ \mathrm{H} & 0.2027 & 4.8591 & -0.0791 \\ \mathrm{~S} & 2.6954 & 0.3161 & 0.0845 \\ \mathrm{C} & 3.3527 & 1.9645 & -0.3470 \\ \mathrm{H} & 3.0864 & 2.2588 & -1.3657 \\ \mathrm{H} & 3.0107 & 2.7288 & 0.3555 \\ \mathrm{H} & 4.4445 & 1.8942 & -0.2748 \\ \mathrm{C} & -3.1160 & -0.3816 & 0.0803 \\ \mathrm{C} & -3.0942 & -1.2583 & 1.2135 \\ \mathrm{C} & -3.0186 & -2.6215 & 1.0504 \\ \mathrm{C} & -3.0191 & -3.1827 & -0.2490 \\ \mathrm{C} & -3.1162 & -2.3491 & -1.3832 \\ \mathrm{C} & -3.1956 & -0.9793 & -1.2162 \\ \mathrm{H} & -3.1024 & -0.8264 & 2.2101 \\ \mathrm{H} & -2.9623 & -3.2934 & 1.9000 \\ \mathrm{H} & -3.1285 & -2.7695 & -2.3820 \\ \mathrm{H} & -3.2790 & -0.3333 & -2.0849 \\ \mathrm{H} & -3.5343 & 0.6101 & 0.2134 \\ \mathrm{O} & -2.9391 & -4.5359 & -0.3011 \\ \mathrm{C} & -2.8961 & -5.1689 & -1.5751 \\ \mathrm{H} & -2.8160 & -6.2389 & -1.3741 \\ \mathrm{H} & -3.8120 & -4.9757 & -2.1488 \\ \mathrm{H} & -2.0252 & -4.8374 & -2.1524\end{array}$

anisole

Etot $=-346.764938$

nbasis $=128$

single point

Etot $=-346.866978$

nbasis $=22$

$\begin{array}{lrrr}\text { C } & -2.2432 & -0.3958 & 1.3649 \\ \text { C } & -1.7313 & -1.1393 & 2.4364 \\ \text { C } & -1.8584 & -2.5240 & 2.4620 \\ \text { C } & -2.5027 & -3.1894 & 1.4070 \\ \text { C } & -3.0185 & -2.4556 & 0.3317 \\ \text { C } & -2.8826 & -1.0625 & 0.3210 \\ \text { H } & -1.2291 & -0.6350 & 3.2581 \\ \text { H } & -1.4666 & -3.1160 & 3.2834 \\ \text { H } & -3.5215 & -2.9509 & -0.4913 \\ \text { H } & -3.2843 & -0.5008 & -0.5187 \\ \text { H } & -2.1388 & 0.6851 & 1.3471 \\ \mathrm{O} & -2.5764 & -4.5484 & 1.5242 \\ \text { C } & -3.2033 & -5.2761 & 0.4826 \\ \text { H } & -3.1432 & -6.3271 & 0.7719 \\ \text { H } & -4.2582 & -4.9920 & 0.3683 \\ \text { H } & -2.6880 & -5.1339 & -0.4769\end{array}$

dimethylaminobenzene adduct

Etot $=-1991.353230$

nbasis $=591$

single point

Etot $=-1991.781798$

nbasis $=987$

$\begin{array}{rrrr}\mathrm{Fe} & 0.5190 & 0.7224 & 0.0000 \\ \mathrm{O} & -1.4051 & 0.9813 & 0.0000 \\ \mathrm{C} & 0.5602 & 4.1483 & 0.0000 \\ \mathrm{~N} & 0.4894 & -0.7425 & 1.4134 \\ \mathrm{C} & 0.5216 & -0.5333 & 2.7745 \\ \mathrm{C} & 0.6378 & -2.0944 & 1.2335 \\ \mathrm{C} & 0.6385 & -1.7954 & 3.4678 \\ \mathrm{H} & 0.6842 & -1.9056 & 4.5444 \\ \mathrm{C} & 0.7221 & -2.7635 & 2.5105 \\ \mathrm{H} & 0.8525 & -3.8314 & 2.6414 \\ \mathrm{~N} & 0.5503 & 2.1511 & 1.4173 \\ \mathrm{C} & 0.5265 & 1.9460 & 2.7685 \\ \mathrm{C} & 0.5553 & 3.5075 & 1.2340 \\ \mathrm{C} & 0.5484 & 4.1859 & 2.5135 \\ \mathrm{H} & 0.5578 & 5.2611 & 2.6422 \\ \mathrm{C} & 0.5289 & 3.2157 & 3.4675 \\ \mathrm{H} & 0.5205 & 3.3264 & 4.5448 \\ \mathrm{~N} & 0.5503 & 2.1511 & -1.4173 \\ \mathrm{C} & 0.5553 & 3.5075 & -1.2340 \\ \mathrm{C} & 0.5265 & 1.9460 & -2.7685 \\ \mathrm{C} & 0.5484 & 4.1859 & -2.5135 \\ \mathrm{H} & 0.5578 & 5.2611 & -2.6422 \\ \mathrm{C} & 0.5289 & 3.2157 & -3.4675 \\ \mathrm{H} & 0.5205 & 3.3264 & -4.5448 \\ \mathrm{~N} & 0.4894 & -0.7425 & -1.4134 \\ \mathrm{C} & 0.6378 & -2.0944 & -1.2335 \\ \mathrm{C} & 0.5216 & -0.5333 & -2.7745 \\ \mathrm{C} & 0.6385 & -1.7954 & -3.4678 \\ \mathrm{H} & 0.6842 & -1.9056 & -4.5444 \\ \mathrm{C} & 0.7221 & -2.7635 & -2.5105 \\ \mathrm{H} & 0.8525 & -3.8314 & -2.6414 \\ \mathrm{C} & 0.5052 & 0.7057 & 3.4031 \\ \mathrm{C} & 0.7008 & -2.7348 & 0.0000 \\ & & & \\ & & & \end{array}$

\begin{tabular}{|c|c|c|c|}
\hline C & 0.5052 & 0.7057 & -3.4031 \\
\hline $\mathrm{H}$ & 0.5173 & 0.7078 & 4.4898 \\
\hline $\mathrm{H}$ & 0.8460 & -3.8120 & 0.0000 \\
\hline $\mathrm{H}$ & 0.5173 & 0.7078 & -4.4898 \\
\hline $\mathrm{H}$ & 0.5671 & 5.2347 & 0.0000 \\
\hline S & 2.8114 & 0.5244 & 0.0000 \\
\hline C & 3.5763 & 2.1873 & 0.0000 \\
\hline $\mathrm{H}$ & 3.2971 & 2.7590 & -0.8902 \\
\hline 1 & 3.2971 & 2.7590 & 0.8902 \\
\hline 11 & 4.6629 & 2.0492 & 0.0000 \\
\hline C & -2.3313 & -0.0201 & 0.0000 \\
\hline C & -2.4198 & -0.8519 & 1.2433 \\
\hline C & -2.6864 & -2.1838 & 1.2423 \\
\hline 0 & -2.7756 & -2.9093 & 0.0000 \\
\hline C & -2.6864 & -2.1838 & -1.2423 \\
\hline C & -2.4198 & -0.8519 & -1.2433 \\
\hline $\mathrm{H}$ & -2.3032 & -0.3124 & 2.1780 \\
\hline $\mathrm{H}$ & -2.7455 & -2.7177 & 2.1823 \\
\hline $\mathrm{H}$ & -2.7455 & -2.7177 & -2.1823 \\
\hline $\mathrm{H}$ & -2.3032 & -0.3124 & -2.1780 \\
\hline $\mathrm{H}$ & -3.3506 & 0.4699 & 0.0000 \\
\hline $\mathrm{N}$ & -2.9449 & -4.2504 & 0.0000 \\
\hline C & -2.9446 & -5.0042 & 1.2606 \\
\hline $\mathrm{H}$ & -3.0984 & -6.0614 & 1.0433 \\
\hline $\mathrm{H}$ & -1.9900 & -4.8872 & 1.7880 \\
\hline $\mathrm{H}$ & -3.7536 & -4.6678 & 1.9175 \\
\hline C & -2.9446 & -5.0042 & -1.2606 \\
\hline $\mathrm{H}$ & -3.0984 & -6.0614 & -1.0433 \\
\hline $\mathrm{H}$ & -3.7536 & -4.6678 & -1.9175 \\
\hline $\mathrm{H}$ & -1.9900 & -4.8872 & -1.7880 \\
\hline
\end{tabular}

dimethylaminobenzene-Compound I transition state

Etot $=-1991.316652$

nbasis $=591$

single point

Etot $=-1991.742069$

nbasis $=987$

\begin{tabular}{|c|c|c|c|}
\hline $\mathrm{Fe}$ & 0.4858 & 0.6510 & 0.0000 \\
\hline 0 & -1.2016 & 0.8769 & 0.0000 \\
\hline C & 0.5555 & 4.0864 & 0.0000 \\
\hline $\mathrm{N}$ & 0.5860 & -0.7754 & 1.4179 \\
\hline C & 0.6142 & -0.5725 & 2.7768 \\
\hline C & 0.6715 & -2.1304 & 1.2312 \\
\hline C & 0.6978 & -1.8392 & 3.4650 \\
\hline $\mathrm{H}$ & 0.7405 & -1.9513 & 4.5412 \\
\hline C & 0.7407 & -2.8060 & 2.5059 \\
\hline $\mathrm{H}$ & 0.8319 & -3.8783 & 2.6301 \\
\hline $\mathrm{N}$ & 0.5995 & 2.0907 & 1.4271 \\
\hline C & 0.5958 & 1.8945 & 2.7817 \\
\hline C & 0.5706 & 3.4470 & 1.2330 \\
\hline C & 0.5614 & 4.1302 & 2.5078 \\
\hline $\mathrm{H}$ & 0.5454 & 5.2061 & 2.6284 \\
\hline C & 0.5789 & 3.1667 & 3.4691 \\
\hline $\mathrm{H}$ & 0.5817 & 3.2843 & 4.5456 \\
\hline $\mathrm{N}$ & 0.5995 & 2.0907 & -1.4271 \\
\hline C & 0.5706 & 3.4470 & -1.2330 \\
\hline C & 0.5958 & 1.8945 & -2.7817 \\
\hline C & 0.5614 & 4.1302 & -2.5078 \\
\hline $\mathrm{H}$ & 0.5454 & 5.2061 & -2.6284 \\
\hline C & 0.5789 & 3.1667 & -3.4691 \\
\hline $\mathrm{H}$ & 0.5817 & 3.2843 & -4.5456 \\
\hline $\mathrm{N}$ & 0.5860 & -0.7754 & -1.4179 \\
\hline C & 0.6715 & -2.1304 & -1.2312 \\
\hline C & 0.6142 & -0.5725 & -2.7768 \\
\hline C & 0.6978 & -1.8392 & -3.4650 \\
\hline $\mathrm{H}$ & 0.7405 & -1.9513 & -4.5412 \\
\hline C & 0.7407 & -2.8060 & -2.5059 \\
\hline $\mathrm{H}$ & 0.8319 & -3.8783 & -2.6301 \\
\hline C & 0.6012 & 0.6591 & 3.4169 \\
\hline C & 0.7073 & -2.7723 & 0.0000 \\
\hline C & 0.6012 & 0.6591 & -3.4169 \\
\hline $\mathrm{H}$ & 0.6170 & 0.6561 & 4.5029 \\
\hline $\mathrm{H}$ & 0.7930 & -3.8550 & 0.0000 \\
\hline $\mathrm{H}$ & 0.6170 & 0.6561 & -4.5029 \\
\hline $\mathrm{H}$ & 0.5358 & 5.1722 & 0.0000 \\
\hline $\mathrm{S}$ & 2.9067 & 0.5554 & 0.0000 \\
\hline C & 3.6240 & 2.2356 & 0.0000 \\
\hline $\mathrm{H}$ & 3.3387 & 2.8002 & -0.8922 \\
\hline $\mathrm{H}$ & 3.3387 & 2.8002 & 0.8922 \\
\hline $\mathrm{H}$ & 4.7130 & 2.1125 & 0.0000 \\
\hline C & -2.9636 & 0.0832 & 0.0000 \\
\hline C & -3.0018 & -0.6587 & 1.2184 \\
\hline C & -2.9415 & -2.0328 & 1.2233 \\
\hline C & -2.8922 & -2.7654 & 0.0000 \\
\hline C & -2.9415 & -2.0328 & -1.2233 \\
\hline C & -3.0018 & -0.6587 & -1.2184 \\
\hline & -3.0358 & -0.1180 & 2.1593 \\
\hline
\end{tabular}

$\begin{array}{rrrr}\mathrm{H} & -2.9183 & -2.5553 & 2.1715 \\ \mathrm{H} & -2.9183 & -2.5553 & -2.1715 \\ \mathrm{H} & -3.0358 & -0.1180 & -2.1593 \\ \mathrm{H} & -3.3227 & 1.1040 & 0.0000 \\ \mathrm{~N} & -2.8057 & -4.1354 & 0.0000 \\ \mathrm{C} & -2.6649 & -4.8603 & 1.2580 \\ \mathrm{H} & -2.5709 & -5.9262 & 1.0470 \\ \mathrm{H} & -1.7744 & -4.5321 & 1.8104 \\ \mathrm{H} & -3.5421 & -4.7170 & 1.9016 \\ \mathrm{C} & -2.6649 & -4.8603 & -1.2580 \\ \mathrm{H} & -2.5709 & -5.9262 & -1.0470 \\ \mathrm{H} & -3.5421 & -4.7170 & -1.9016 \\ \mathrm{H} & -1.7744 & -4.5321 & -1.8104\end{array}$

dimethylaminobenzene

Etot $=-366.207699$

nbasis $=148$

single point

Etot $=-366.313521$

nbasis $=264$

$\begin{array}{rrrr}\text { C } & 0.2832 & 2.5930 & 0.0000 \\ \text { C } & 0.0390 & -0.2399 & 0.0000 \\ \text { C } & 0.0907 & 0.4934 & -1.2083 \\ \text { C } & 0.2157 & 1.8814 & 1.1991 \\ \text { C } & 0.0907 & 0.4934 & 1.2083 \\ \mathrm{C} & 0.2157 & 1.8814 & -1.1991 \\ \mathrm{H} & 0.3761 & 3.6751 & 0.0000 \\ \mathrm{H} & 0.2537 & 2.4095 & 2.1491 \\ \mathrm{H} & 0.0307 & -0.0172 & 2.1623 \\ \mathrm{~N} & -0.0505 & -1.6329 & 0.0000 \\ \mathrm{H} & 0.0307 & -0.0172 & -2.1623 \\ \mathrm{H} & 0.2537 & 2.4095 & -2.1491 \\ \mathrm{C} & -0.3665 & -2.3199 & 1.2419 \\ \mathrm{H} & -0.3664 & -3.3976 & 1.0631 \\ \mathrm{H} & 0.3922 & -2.1145 & 2.0058 \\ \mathrm{H} & -1.3494 & -2.0372 & 1.6549 \\ \mathrm{C} & -0.3665 & -2.3199 & -1.2419 \\ \mathrm{H} & -0.3664 & -3.3976 & -1.0631 \\ \mathrm{H} & -1.3494 & -2.0372 & -1.6549 \\ \mathrm{H} & 0.3922 & -2.1145 & -2.0058\end{array}$

benzene epoxide adduct

Etot $=-1857.386178$

nbasis $=539$

single point

Etot $=-1857.776140$

nbasis $=891$

$\begin{array}{rrrr}\text { Fe } & -0.1735 & -1.5057 & -1.7836 \\ \mathrm{O} & -2.0497 & -0.4960 & -0.9819 \\ \mathrm{C} & -0.3720 & 1.0122 & -4.1093 \\ \mathrm{~N} & 0.5324 & -1.6603 & 0.0972 \\ \mathrm{C} & 1.3273 & -0.7412 & 0.7405 \\ \mathrm{C} & 0.4661 & -2.7493 & 0.9338 \\ \mathrm{C} & 1.7464 & -1.2528 & 2.0252 \\ \mathrm{H} & 2.3770 & -0.7177 & 2.7242 \\ \mathrm{C} & 1.2089 & -2.4979 & 2.1463 \\ \mathrm{H} & 1.3079 & -3.2006 & 2.9642 \\ \mathrm{~N} & 0.5095 & 0.3790 & -1.9090 \\ \mathrm{C} & 1.3062 & 1.0182 & -0.9900 \\ \mathrm{C} & 0.3481 & 1.2638 & -2.9487 \\ \mathrm{C} & 1.0610 & 2.4904 & -2.6788 \\ \mathrm{H} & 1.0925 & 3.3437 & -3.3448 \\ \mathrm{C} & 1.6611 & 2.3354 & -1.4659 \\ \mathrm{H} & 2.2848 & 3.0370 & -0.9261 \\ \mathrm{~N} & -1.0359 & -1.2923 & -3.5829 \\ \mathrm{C} & -1.0044 & -0.1885 & -4.4049 \\ \mathrm{C} & -1.6994 & -2.2685 & -4.2889 \\ \mathrm{C} & -1.6773 & -0.4716 & -5.6500 \\ \mathrm{H} & -1.7887 & 0.2334 & -6.4643 \\ \mathrm{C} & -2.1024 & -1.7644 & -5.5805 \\ \mathrm{H} & -2.6393 & -2.3395 & -6.3247 \\ \mathrm{~N} & -0.9606 & -3.3551 & -1.6038 \\ \mathrm{C} & -0.8247 & -4.2265 & -0.5520 \\ \mathrm{C} & -1.6151 & -4.0543 & -2.5887 \\ \mathrm{C} & -1.9118 & -5.3957 & -2.1398 \\ \mathrm{H} & -2.4214 & -6.1447 & -2.7332 \\ \mathrm{C} & -1.4246 & -5.5014 & -0.8729 \\ \mathrm{H} & -1.4463 & -6.3565 & -0.2088 \\ \mathrm{C} & 1.6941 & 0.5009 & 0.2398 \\ \mathrm{C} & -0.1731 & -3.9463 & 0.6422 \\ \mathrm{C} & -1.9618 & -3.5561 & -3.8371 \\ \mathrm{H} & 2.3321 & 1.1210 & 0.8629 \\ \mathrm{H} & -0.1303 & -4.7341 & 1.3887 \\ \mathrm{H} & -2.4849 & -4.2250 & -4.5145 \\ \mathrm{H} & -0.4134 & 1.7994 & -4.8565 \\ \mathrm{~S} & 1.7031 & -2.4140 & -2.5633 \\ \mathrm{C} & 2.2659 & -1.4809 & -4.0325 \\ \mathrm{H} & 1.5270 & -1.5139 & -4.8369 \\ & & & \end{array}$




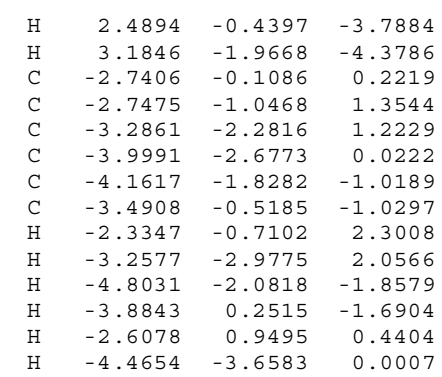

benzene epoxide transition state Etot $=-1857.352887$

nbasis $=539$

single point

Etot $=-1857.742495$

nbasis $=89$

\begin{tabular}{rrrr}
$\mathrm{Fe}$ & -0.0913 & 0.0032 & -0.0214 \\
$\mathrm{O}$ & -0.1344 & 0.1590 & 1.9096 \\
$\mathrm{C}$ & 3.3007 & 0.0336 & 0.4720 \\
$\mathrm{~N}$ & -1.5109 & -1.4286 & -0.2112 \\
$\mathrm{C}$ & -1.2818 & -2.7897 & -0.2756 \\
$\mathrm{C}$ & -2.8363 & -1.2594 & -0.5217 \\
$\mathrm{C}$ & -2.5067 & -3.4872 & -0.5917 \\
$\mathrm{H}$ & -2.5937 & -4.5611 & -0.7017 \\
$\mathrm{C}$ & -3.4713 & -2.5377 & -0.7442 \\
$\mathrm{H}$ & -4.5142 & -2.6711 & -1.0050 \\
$\mathrm{~N}$ & 1.3335 & -1.4047 & 0.2035 \\
$\mathrm{C}$ & 1.1561 & -2.7606 & 0.1402 \\
$\mathrm{C}$ & 2.6766 & -1.2060 & 0.4122 \\
$\mathrm{C}$ & 3.3622 & -2.4757 & 0.5021 \\
$\mathrm{H}$ & 4.4266 & -2.5924 & 0.6639 \\
$\mathrm{C}$ & 2.4184 & -3.4418 & 0.3274 \\
$\mathrm{H}$ & 2.5450 & -4.5172 & 0.3160 \\
$\mathrm{~N}$ & 1.3236 & 1.4321 & 0.1041 \\
$\mathrm{C}$ & 2.6668 & 1.2610 & 0.3114 \\
$\mathrm{C}$ & 1.1237 & 2.7790 & -0.0110 \\
$\mathrm{C}$ & 3.3400 & 2.5408 & 0.3198 \\
$\mathrm{H}$ & 4.4052 & 2.6773 & 0.4571 \\
$\mathrm{C}$ & 2.3804 & 3.4851 & 0.1158 \\
$\mathrm{H}$ & 2.4923 & 4.5602 & 0.0517 \\
$\mathrm{~N}$ & -1.5357 & 1.4042 & -0.2707 \\
$\mathrm{C}$ & -2.8633 & 1.2102 & -0.5213 \\
$\mathrm{C}$ & -1.3308 & 2.7637 & -0.3416 \\
$\mathrm{C}$ & -2.5803 & 3.4426 & -0.6111 \\
$\mathrm{H}$ & -2.6893 & 4.5147 & -0.7182 \\
$\mathrm{C}$ & -3.5331 & 2.4766 & -0.7239 \\
$\mathrm{H}$ & -4.5880 & 2.5890 & -0.9428 \\
$\mathrm{C}$ & -0.0561 & -3.4084 & -0.0921 \\
$\mathrm{C}$ & -3.4869 & -0.0343 & -0.6140 \\
$\mathrm{C}$ & -0.1072 & 3.4018 & -0.2150 \\
$\mathrm{H}$ & -0.0337 & -4.4928 & -0.1563 \\
$\mathrm{H}$ & -4.5438 & -0.0469 & -0.8662 \\
$\mathrm{H}$ & -0.3329 & -0.6257 & 3.8047 \\
$\mathrm{H}$ & -4.8401 & 0.2538 & 2.3501 \\
$\mathrm{H}$ & -0.1040 & 4.4851 & -0.3002 \\
$\mathrm{H}$ & 4.3760 & 0.0433 & 0.6265 \\
$\mathrm{~S}$ & 0.0776 & -0.0532 & -2.3050 \\
$\mathrm{C}$ & 1.8317 & -0.0882 & -2.8228 \\
$\mathrm{H}$ & 2.3614 & 0.8200 & -2.5220 \\
$\mathrm{H}$ & 2.3542 & -0.9608 & -2.4201 \\
$\mathrm{H}$ & 1.8398 & -0.1494 & -3.9160 \\
$\mathrm{C}$ & -0.9712 & -0.3228 & 2.9585 \\
$\mathrm{C}$ & -1.9253 & -1.4434 & 2.6616 \\
$\mathrm{C}$ & -3.2633 & -1.2286 & 2.5244 \\
$\mathrm{C}$ & -3.7817 & 0.1012 & 2.5452 \\
$\mathrm{H}$ & -2.9726 & 1.2015 & 2.8403 \\
$\mathrm{H}$ & -1.5395 & 0.9905 & 3.1553 \\
& -3.3883 & -2.4493 & 2.6183 \\
& 2.2045 & 2.3225 \\
\hline
\end{tabular}

ketone adduct

Etot $=-1857.435432$

nbasis $=539$

single point

Etot $=-1857.825611$

nbasis $=891$

$\begin{array}{rrrr}\text { Fe } & 0.4499 & 0.3095 & -0.0374 \\ \mathrm{O} & -1.7384 & 0.1131 & -0.1603 \\ \mathrm{C} & -0.0309 & 3.7070 & 0.0375 \\ \mathrm{~N} & 0.4821 & -1.1724 & 1.3406 \\ \mathrm{C} & 0.4938 & -1.0031 & 2.7038\end{array}$

\begin{tabular}{|c|c|c|c|}
\hline C & 0.6446 & -2.5195 & 1.1260 \\
\hline C & 0.6513 & -2.2787 & 3.3647 \\
\hline $\mathrm{H}$ & 0.6923 & -2.4145 & 4.4382 \\
\hline C & 0.7413 & -3.2203 & 2.3853 \\
\hline H & 0.8742 & -4.2902 & 2.4858 \\
\hline N & 0.1949 & 1.6825 & 1.4038 \\
\hline C & 0.2181 & 1.4519 & 2.7575 \\
\hline C & 0.0172 & 3.0369 & 1.2531 \\
\hline C & -0.0780 & 3.6767 & 2.5446 \\
\hline $\mathrm{H}$ & -0.2179 & 4.7396 & 2.6967 \\
\hline C & 0.0515 & 2.6937 & 3.4781 \\
\hline $\mathrm{H}$ & 0.0374 & 2.7813 & 4.5574 \\
\hline 10 & 0.3081 & 1.7612 & -1.4163 \\
\hline C & 0.1208 & 3.1054 & -1.2051 \\
\hline C & 0.4380 & 1.6066 & -2.7743 \\
\hline C & 0.1342 & 3.8179 & -2.4620 \\
\hline $\mathrm{H}$ & 0.0098 & 4.8887 & -2.5643 \\
\hline C & 0.3365 & 2.8882 & -3.4357 \\
\hline $\mathrm{H}$ & 0.4101 & 3.0358 & -4.5060 \\
\hline $\mathrm{N}$ & 0.5796 & -1.0948 & -1.4874 \\
\hline C & 0.7286 & -2.4536 & -1.3358 \\
\hline C & 0.6867 & -0.8504 & -2.8354 \\
\hline C & 0.8843 & -2.0884 & -3.5531 \\
\hline $\mathrm{H}$ & 0.9989 & -2.1651 & -4.6272 \\
\hline C & 0.9067 & -3.0840 & -2.6231 \\
\hline $\mathrm{H}$ & 1.0459 & -4.1471 & -2.7745 \\
\hline C & 0.3642 & 0.2109 & 3.3669 \\
\hline C & 0.7408 & -3.1268 & -0.1203 \\
\hline C & 0.6189 & 0.3994 & -3.4381 \\
\hline $\mathrm{H}$ & 0.3810 & 0.1897 & 4.4528 \\
\hline $\mathrm{H}$ & 0.8766 & -4.2041 & -0.1447 \\
\hline $\mathrm{H}$ & 0.7173 & 0.4364 & -4.5192 \\
\hline $\mathrm{H}$ & -0.1698 & 4.7839 & 0.0618 \\
\hline $\mathrm{S}$ & 2.6860 & 0.3866 & 0.0643 \\
\hline C & 3.2301 & 2.1328 & 0.0561 \\
\hline $\mathrm{H}$ & 2.9399 & 2.6434 & -0.8659 \\
\hline $\mathrm{H}$ & 2.8337 & 2.6823 & 0.9142 \\
\hline $\mathrm{H}$ & 4.3236 & 2.1192 & 0.1221 \\
\hline C & -2.4986 & -0.8457 & 0.0281 \\
\hline C & -2.9733 & -1.2077 & 1.3539 \\
\hline C & -3.8074 & -2.2677 & 1.5217 \\
\hline C & -4.2747 & -3.0852 & 0.4136 \\
\hline C & -3.8809 & -2.8180 & -0.8474 \\
\hline C & -2.9468 & -1.6904 & -1.1526 \\
\hline $\mathrm{H}$ & -2.6179 & -0.6094 & 2.1864 \\
\hline $\mathrm{H}$ & -4.1434 & -2.5268 & 2.5235 \\
\hline $\mathrm{H}$ & -4.2250 & -3.4262 & -1.6807 \\
\hline $\mathrm{H}$ & -2.0261 & -2.0742 & -1.6219 \\
\hline $\mathrm{H}$ & -3.3738 & -1.0190 & -1.9127 \\
\hline $\mathrm{H}$ & -4.9429 & -3.9145 & 0.6263 \\
\hline
\end{tabular}

ketone transition state Etot $=-1857.363619$

nbasis $=539$

single point

Etot $=-1857.759117$

nbasis $=891$

$\begin{array}{rrrr}\mathrm{Fe} & 0.3451 & 0.3270 & -0.0123 \\ \mathrm{O} & -1.6714 & 0.4393 & -0.0105 \\ \mathrm{C} & 0.2155 & 3.7538 & 0.0022 \\ \mathrm{~N} & 0.3499 & -1.1379 & 1.3969 \\ \mathrm{C} & 0.4038 & -0.9343 & 2.7570 \\ \mathrm{C} & 0.5065 & -2.4911 & 1.2097 \\ \mathrm{C} & 0.5481 & -2.1973 & 3.4433 \\ \mathrm{H} & 0.6134 & -2.3097 & 4.5186 \\ \mathrm{C} & 0.6169 & -3.1620 & 2.4830 \\ \mathrm{H} & 0.7483 & -4.2298 & 2.6073 \\ \mathrm{~N} & 0.3023 & 1.7498 & 1.4090 \\ \mathrm{C} & 0.3206 & 1.5412 & 2.7620 \\ \mathrm{C} & 0.2467 & 3.1081 & 1.2325 \\ \mathrm{C} & 0.2380 & 3.7803 & 2.5136 \\ \mathrm{H} & 0.2040 & 4.8545 & 2.6465 \\ \mathrm{C} & 0.2851 & 2.8071 & 3.4640 \\ \mathrm{H} & 0.2984 & 2.9147 & 4.5415 \\ \mathrm{~N} & 0.3024 & 1.7599 & -1.4209 \\ \mathrm{C} & 0.2490 & 3.1170 & -1.2331 \\ \mathrm{C} & 0.3250 & 1.5623 & -2.7758 \\ \mathrm{C} & 0.2449 & 3.7996 & -2.5083 \\ \mathrm{H} & 0.2133 & 4.8749 & -2.6320 \\ \mathrm{C} & 0.2933 & 2.8343 & -3.4669 \\ \mathrm{H} & 0.3100 & 2.9515 & -4.5434 \\ \mathrm{~N} & 0.3517 & -1.1289 & -1.4333 \\ \mathrm{C} & 0.5076 & -2.4840 & -1.2555 \\ \mathrm{C} & 0.4084 & -0.9145 & -2.7915 \\ \mathrm{C} & 0.5544 & -2.1725 & -3.4871 \\ \mathrm{H} & 0.6225 & -2.2765 & -4.5630 \\ \mathrm{C} & 0.6208 & -3.1451 & -2.5336 \\ \mathrm{H} & 0.7526 & -4.2118 & -2.6661\end{array}$

$\begin{array}{rrr}0.3642 & 0.3006 & 3.3925 \\ 0.5716 & -3.1270 & -0.0248 \\ 0.3693 & 0.3267 & -3.4165 \\ 0.4022 & 0.2995 & 4.4784 \\ 0.7154 & -4.2036 & -0.0278 \\ 0.4097 & 0.3354 & -4.5022 \\ 0.1778 & 4.8395 & 0.0062 \\ 2.6148 & 0.2186 & -0.0120 \\ 3.3105 & 1.9111 & 0.0029 \\ 3.0191 & 2.4751 & -0.8877 \\ 3.0041 & 2.4655 & 0.8943 \\ 4.4012 & 1.8092 & 0.0117 \\ -2.3767 & -0.6608 & 0.0071 \\ -2.6521 & -1.3737 & 1.2707 \\ -3.1370 & -2.6620 & 1.2805 \\ -3.3388 & -3.3592 & 0.0678 \\ -3.1134 & -2.7505 & -1.1666 \\ -2.6005 & -1.4556 & -1.2188 \\ -2.4684 & -0.8171 & 2.1834 \\ -3.3284 & -3.1628 & 2.2252 \\ -3.2907 & -3.2947 & -2.0893 \\ -2.4175 & -0.9360 & -2.1530 \\ -3.5325 & -0.4311 & -0.2462 \\ -3.7068 & -4.3811 & 0.0994\end{array}$

